\title{
Journal of Pest Science \\ The potential of nanobiopesticide based on zein nanoparticles and neem oil for enhanced control of agricultural pests \\ --Manuscript Draft--
}

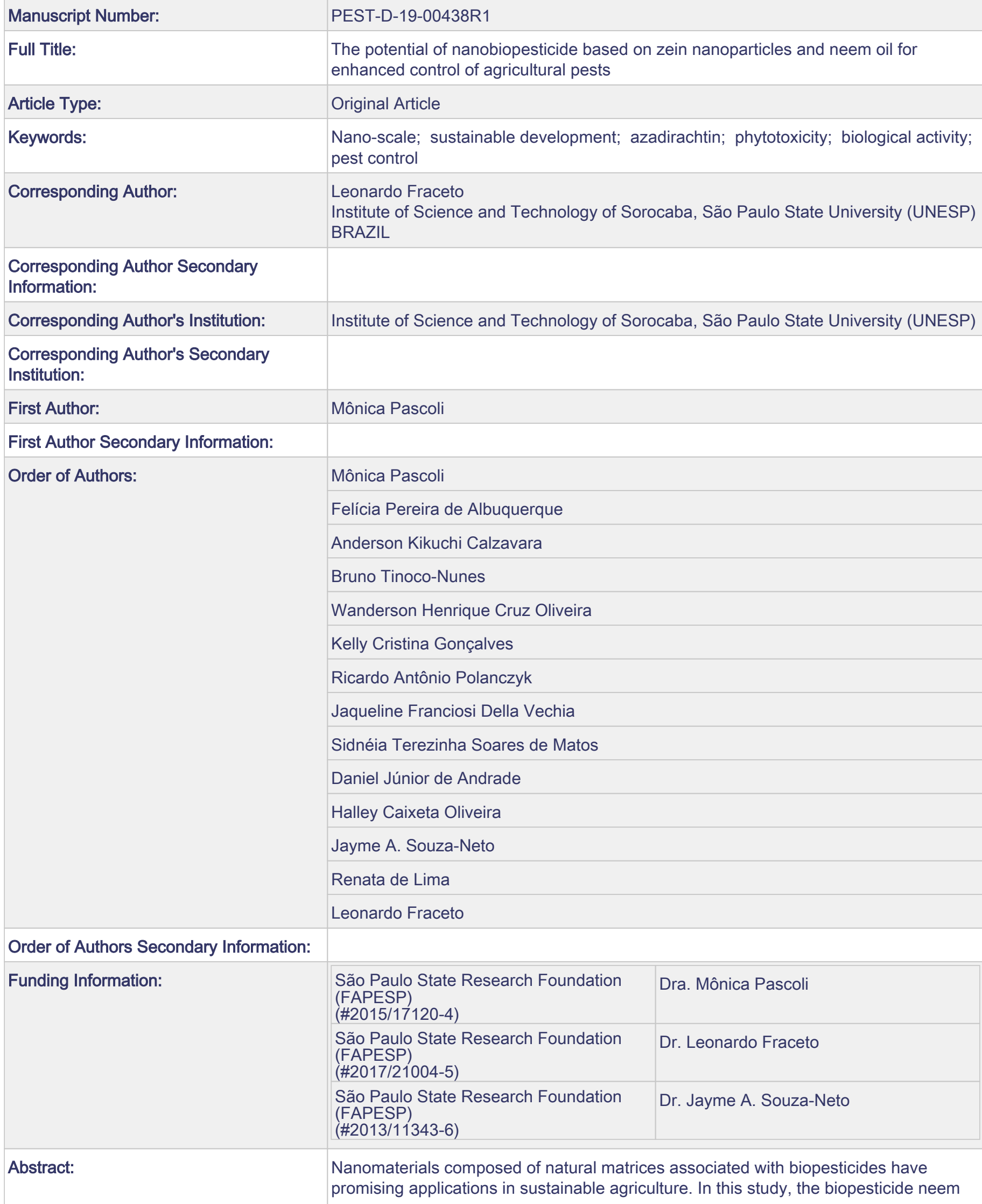


oil was encapsulated in zein nanoparticles in order to improve its stability and efficiency. Assays of phytotoxicity (using Phaseolus vulgaris) and biological activity against three pests (Acanthoscelides obtectus, Bemisia tabaci, and Tetranychus urticae) were also performed. The neem oil-loaded zein nanoparticles presented $198 \pm$ $16 \mathrm{~nm}$, polydispersity index of around 0.2 , satisfactory physicochemical stability, together with high encapsulation efficiency $(>80 \%)$. Pre- and post-emergence treatments using this new system did not cause any phytotoxic effects towards $\mathrm{P}$. vulgaris. The neem oil nanobiopesticide exhibited mortality effects on $B$. tabaci and $T$. urticae, while the effect against A. obtectus was significantly increased, compared to plain neem oil. The results of the characterization, toxicity, and biological activity studies showed the promising potential of these neem oil-loaded zein nanoparticles for use in pest management in sustainable agriculture after the required toxicological assessments.

\section{Response to Reviewers:}

Sorocaba, November 26th 2019.

Dear Prof. Michael Traugott

Editor in Chief

Journal of Pest Science

The authors are very thankful to the Editor and Reviewers for their valuable comments and remarks regarding the manuscript. We have addressed all comments and suggestions adequately. The requested alterations/corrections have been inserted directly into the manuscript (significant changes are highlighted in blue) and are described below.

Yours sincerely,

Leonardo Fernandes Fraceto

Corresponding author

E-mail: leonardo.fraceto@unesp.br

Editor-in-Chief:

Please consider the following points when revising your manuscript:

Title: rephrase the title that it does inform on the most significant findings of your study, i.e. the concrete take home message in a nutshell

Answer: Thank you very much for your suggestion. We modified the manuscript title for "The potential of nanobiopesticide based on zein nanoparticles and neem oil for enhanced control of agricultural pests"

\section{Subject Editor:}

Both reviewers see merit in this paper, but both have identified a number of weaknesses, especially in the general presentation. I recommend that the authors be provided the opportunity to address these concerns, and submit a substantially revised and improved manuscript based on the reviewers' comments and suggestions. Answer: Thank you very much for your careful evaluation and suggestions for the improvement of our manuscript. We revised and modified it based on the reviewers' comments and describe each adjustment below.

\section{Reviewer \#1:}

Reviewer: The manuscript entitled "NANOBIOPESTICIDE BASED ON ZEIN NANOPARTICLES AND NEEM OIL: A STUDY USING TARGET AND NONTARGET ORGANISMS" seems an interesting and novel study unless the authors seriously address and incorporate the following suggestions to improve their MS for broad readership.

Answer: Thank you for your comments. We modified the manuscript in order to make it more enlightening for Journal of Pest Science readers. We answer each comment individually.

Major Concerns:

Reviewer: The text remains to be improved to become of acceptable standard. In general, the quality of the English must be improved, preferably by consulting language editing services. 
Answer: Thank you for your comment. English was reviewed by a native speaker. Please verify the revised version of the manuscript.

Reviewer: Consider to reanalyze mortality data using repeated measures ANOVA or survival analysis (preferably).

Answer: Thank you for your comment. We have reanalyzed mortality data using repeated measures ANOVA. Please verify the revised version of the manuscript.

Reviewer: Lack of detailed statistical analysis in the results sections.

Answer: Thank you for your comment. We have inserted this information in all figure captions and in the results section. Please verify the revised version of the manuscript.

Reviewer: Results section is not appropriately prepared.

Answer: Thank you for your comment. We have abbreviated the results section. Please verify the revised version of the manuscript.

Reviewer: Discussion section needs to be re-written.

Answer: Thank you for your comment. We have improved the discussion section.

Please verify the revised version of the manuscript.

Reviewer: Lack of conclusion of the study.

Answer: Thank you for your comment. We have rewritten the conclusion. Please verify the revised version of the manuscript.

Reviewer: Lack of necessary details in the figures.

Answer: Thank you for your comment. We have improved the details in the figures.

Please verify the revised version of the manuscript.

Reviewer: Statistical analysis issues

Answer: Thank you for your comment. We have reviewed the statistical analysis.

Please verify the revised version of the manuscript

Minor Concerns:

Reviewer: Line 57-58. Syntax error, I will suggest to rewrite the sentence for clarity Answer: Thank you very much for your suggestion. The key message was reviewed and rewritten (p. 3, I. 55). Please verify the revised version of the manuscript.

Reviewer: Line 69-71. 67,000 pest species ae not under the mentioned citation. This number was calculated long before. I will suggest to correct the citation.

Answer: Thank you for your comment. We apologize for the incorrect citation. Now, we cited Ross and Lembi, 1985 (p. 4, I. 70). Please verify the revised version of the manuscript.

Reviewer: Line 139-142. I will suggest to rewrite the sentence for clarity. In addition, currently the sentence lacks detailed objectives of the study. I will suggest the authors to provide the objectives of the study.

Answer: Thank you for your comment. We have rewritten the objectives of the study ( $p$. 5, I. 110). Please verify the revised version of the manuscript.

Reviewer: Line 144-157. I will suggest to provide the Catalogue number of each material purchased to complete the current study.

Answer: Thank you for your comment. We have added the catalogue number of each material in the revised version. Please verify the revised version of the manuscript.

Reviewer: Line 164. I will suggest to use standard unit for centrifugation instead of * Answer: Thank you for your comment. We have added the standard unit for centrifugation (p.7, I.141)

Reviewer: Line 172-173. Grammatical error. I will suggest to rewrite the sentence. Answer: Thank you for your comment. We have rewritten the sentence (p. 7, I. 149). Please verify the revised version of the manuscript.

Reviewer: Line 193-195. Something is missing in the following sentence "The images 
were collected using a sCMOS camera and were processed using NanoSight v. 2.3 software Grillo et al. (2014). For these analyses, the samples were diluted 1000 times." Answer: Thank you for your comment. We have corrected the citation for a better understanding of the sentence (p. 8, I. 173).

Reviewer: Line 225. Chlorophyll a fluorescence. Please recheck "a" Answer: Thank you for your comment. The correct form used in plant physiological studies is "a" in italic. Please verify the revised version of the manuscript.

Reviewer: Line 381-383. Results section does not need to add citation. I will suggest to delete here and throughout results.

Answer: Thank you for your comment. We have deleted all citations from the Results section. Please verify the revised version of the manuscript.

Reviewer: Line 393-394. Awkward arrangement of the sentence.

Answer: Thank you for your comment. We have modified the sentence for "The nanoparticle concentration evaluated by NTA (Figure 1C) showed significant fluctuations during the 90 days of storage $(F=172.5, D F=5, P<0.0001) . "(p .17, \mathrm{l}$. 370). Please verify the revised version of the manuscript.

Reviewer: Figure 1. Firstly, some of the bars lack lettering. Secondly, some bars following pattern of ascending and other following decending order to lettering. I will suggest to follow same rule which is scientifically acceptable. Thirdly, in case of Figure $1 \mathrm{~b}$, polydispersity index (line), I could not see the SE bars.

Answer: Thank you for your comment. We have modified the way we indicate data significance. It is unable to see some SE bars because their values are too small. Please verify the revised version of the manuscript.

Reviewer: Line 415-417. No need to add citation in this section.

Answer: Thank you for your comment. We have deleted all citations from the Results section. Please verify the revised version of the manuscript.

Reviewer: Line 441. The authors did not provide the lettering. Without lettering hard to understand the level of significant differences.

Answer: Thank you for your comment. We have modified the way we indicate data significance. Please verify the revised version of the manuscript.

Reviewer: Line 462. Results section does not need citation here and elsewhere in the MS.

Answer: Thank you for your comment. We have deleted all citations from the Results section. Please verify the revised version of the manuscript.

Reviewer: Line 464. Stands for what?

Answer: Thank you for your comment. We have completed the idea "indicating that the new system provided greater efficiency against this bean pest, compared to the traditional neem oil" (p. 22, I. 445). Please verify the revised version of the manuscript.

Reviewer: Figure $3 a$, authors mentioned that letters $a, b$, and $c$ indicate significant difference relative to the control. Firstly, i could not find "b" in the lettering, i could only find "a" and "c". Secondly, Randomly providing lettering is not acceptable. I will sugest to provide the original letter(s) as a results of mean comparison test. Thirdly, i will suggest the authors to provide name of the means comparison test along with type of analysis.

Answer: Thank you for your comment. We have modified the way we indicate data significance and inserted more information in figure caption (p. 24, I. 467). Please verify the revised version of the manuscript.

Reviewer: I disagree with the authors regarding Figure 4 analysis. I suggest to apply survival analysis and their curves otherwise repeated measures ANOVA. Answer: Thank you for your comment. We have reanalyzed mortality data using repeated measures ANOVA. Please verify the revised version of the manuscript.

Reviewer: Line 551-579. I will suggest the authors to provide the details of the analysis such as $\mathrm{F}$ value, df and $\mathrm{P}$-value, here and throughout the manuscript. 
Answer: Thank you for your comment. We have inserted the requested information throughout the manuscript. Please verify the revised version.

Reviewer: Figure 5. I do not agree with the analysis. I will suggest to apply repeated measures ANOVA or survival analysis and their curves.

Answer: Thank you for your comment. We would like to explain that in Figure 5, there is no need to perform repeated measurements ANOVA because our data do not represent measurements over time of the same sample. In fact, different leaves were collected on different days after the application of the treatments to perform the test, and not a single leaf was collected and analyzed 1, 6 and 12 days latter. We have modified the figure caption for better understanding. Please verify the revised version of the manuscript.

Reviewer: Line 591-801. Firstly, discussion section seems like a review of literature and many of the sentenses are awkwardly placed. I will suggest to rewrite the whole sentense. Secondly, hard to get the idea due to linguistic and syntax errors.

Answer: Thank you for your comment. We have abbreviated the discussion and some cited studies were deleted. Please verify the revised version of the manuscript.

Reviewer: Line 592-600. The opening paragraph of the discussion section is not appropriately written. I will suggest to rewrite this section.

Answer: Thank you for your comment. We have rewritten the discussion. Please verify the revised version of the manuscript.

Reviewer: Line 553-554. The sentense is awkwardly placed.

Answer: Thank you for your comment. We have deleted the indicated sentence.

Reviewer: Line 801. I will suggest the authors to provide a comprehensive conclusion of the study.

Answer: Thank you for your comment. We have rewritten the conclusion. Please verify the revised version of the manuscript.

Reviewer \#2:

Reviewer: I have read the manuscript entitled " Nanobiopesticide based on zein nanoparticles and neem oil: a study using target and nontarget organisms". The manuscript presents a well-written and appropriately analyzed series of experiments to determine the pesticidal and biological activity of neem oil-loaded zein nanoparticles against three pests (Acanthoscelides obtectus, Bemisia tabaci, and Tetranychus urticae), in addition to the phytotoxic effects of these nanoparticles using Phaseolus vulgaris.

Overall, the manuscript is generally clear and concise report of a well-executed study. The objectives are clear; the experiments are pertinent and follow a logical reasoning; the main findings of the study are convincing and the conclusion is appropriate. The paper is clearly organized and the contribution is interesting and falls within the scope of the journal. The work is generally well written, except for certain parts of the manuscript, where a good technical editing to improve English and grammar is needed. I have made a number of suggestions and comments to improve the overall clarity and quality of the manuscript, which represent major issues. I think that this study is could be accepted for publication after considering the following major revisions.

Answer: Thank you for your comments. We are happy for your valuable comments, which have greatly improved the quality of the manuscript. We have checked and reviewed all points commented here, as well as the comments from reviewer \#1. English was reviewed by a native speaker. Please verify the revised version of the manuscript.

Abstract

Reviewer: 1. L26 -29 "Nanotechnology has been widely explored with the aim of achieving a new revolution in crop protection, especially considering the development 
of improved biopesticides that offer increased stability and efficiency of the natural active compounds, while reducing the possible adverse effects on nontarget organisms." Long sentence. This sentence should be abbreviated and transferred to the introduction.

Answer: Thank you for your comment. We have deleted the sentence.

Reviewer: 2. L40-43 " The results of the characterization, toxicity, and biological activity studies showed the promising potential of these neem oil-loaded zein nanoparticles for use in pest management in sustainable agriculture." Please recast to "The results of the characterization, toxicity, and biological activity studies showed the promising potential of these neem oil-loaded zein nanoparticles for use in pest management in sustainable agriculture after the required toxicological assessments."

Answer: Thank you for your comment. We have rewritten the sentence as suggested (p. 2, I. 37). Please verify the revised version of the manuscript.

Reviewer: 3. Please consider some of the obtained data in the abstract for clarity Answer: Thank you for your comment. We have inserted some data in abstract section, (p. 2, I. 32).

\section{Key Message}

Reviewer: Please consider the journal guidelines in editing the key message.

Answer: Thank you for your comment. The key message was reviewed and rewritten (p. 3, I. 45).

Introduction

Reviewer: 1. Two long. Please abbreviate.

Answer: Thank you for your comment. We have abbreviated the introduction. Please verify the revised version of the manuscript.

Reviewer: 2. Line 126-131 " The aim of the present study was to investigate the effects of neem oil-loaded zein nanoparticles on target organisms, in order to evaluate the potential of this system as a nanobiopesticide. Its biological efficacy was evaluated against three species of agricultural pest: i) the bean weevil Acanthoscelides obtectus (Say), ii) the whitefly Bemisia tabaci (Gennadius), and iii) the two-spotted spider mite (T. urticae)."

Please recast to " The aim of the present study was to investigate the biological efficacy of neem oil-loaded zein nanoparticles against three species of agricultural pest: i) the bean weevil A. obtectus (Say), ii) the whitefly B. tabaci (Gennadius), and iii) the two-spotted spider mite (T. urticae)." as target organisms. The phytotoxic effects of these nanoparticles against $P$. vulgaris was also evaluated.

Answer: Thank you for your comment. We have rewritten the sentence as suggested (p. 5, I. 110).

\section{Materials and Methods}

Reviewer: 1. The authors consider this section as: 2.1. Materials, while 2.2. section is not found. Please consider this section as a main title; 2 . Materials and methods, then subtitles start with 2.1. Chemicals 2.2. Test organisms. The authors are kindly asked to decrease the subtitles.

Answer: Thank you for your comment. We considered section 2 as Materials and Methods, modified the section 2.1 for Supplies and section 2.2 as the Preparation of neem oil-loaded zein nanoparticles. Please verify the revised version of the manuscript.

Reviewer: 2. Please consider the label Purity of the chemicals used.

Answer: Thank you for your comment. We have added the requested information. Please verify the revised version of the manuscript, (p. 6, I. 122).

Reviewer: 3. Lin 158: consider Firstly, instead of First.

Answer: Thank you for your comment. We have corrected the word (p. 7, I. 139).

Reviewer: 4. Lin 168-170: "This concentration was chosen since in agriculture, neem oil is used at concentrations of between 4 and $6 \mathrm{mg} / \mathrm{mL}$. Bad phraseology. Please rewrite.

Answer: Thank you for your comments. We have rewritten the sentence "In field, neem 
oil is used at concentrations between 4 and $6 \mathrm{mg} / \mathrm{mL}$; thus, an intermediate concentration was chosen for the formulation". Please verify the revised version of the manuscript, (p. 7, I. 149).

Reviewer: 5. Lines 171-174: " Labeled nanoparticles, with and without neem oil, were also prepared with addition of rhodamine (18:1 Liss Rhod PE) in the zein solution $(0.05 \% \mathrm{~m} / \mathrm{m}$, relative to the polymer), in order to investigate the interaction between the bean weevils and the formulation". Please support with a convenient reference.

Answer: Thank you for your comment. We have inserted the requested reference in the manuscript (Gott et al. 2014), (p. 7, I. 155).

Reviewer: 6. Lin 181-182: " The same equipment was used to determine the zeta potential, according to the microelectrophoresis method". Please support with a convenient reference.

Answer: Thank you for your comment. We have inserted the requested reference in the manuscript (Grillo et al. 2012), (p. 8, I. 163).

Reviewer: 7. Lines 191-192: Consider (Grillo et al. 214) instead of Grillo et al. (2014). Answer: Thank you for your comment. We have corrected the reference (p. 8, I. 173). Please verify the revised version of the manuscript.

Reviewer: 8. Lin 199: consider (Dubhashi et al. 2013) instead of (Dubhashi et al., 2013).

Answer: Thank you for your comment. We have corrected the reference (p. 8, I. 180).

Reviewer: 9. Lines 278-279: consider Ten unsexed adults (1 to 5 day old) of A. obtectus were placed in each vial istead of: Ten 1 to 5 day old adults of $A$. obtectus (unsexed) were placed in each vial.

Answer: Thank you for your comment. We have replaced the sentence for "Ten unsexed adults ( 1 to 5 day old) of A. obtectus were placed in each vial”, (p. 12, I. 262). Please verify the revised version of the manuscript.

Reviewer: 10. Lines 279-280: "The experiment was carried out using concentrations equivalent to $1.35,2.7,5.4,10.8$, and $21.6 \mathrm{mg}$ of azadirachtin per $\mathrm{kg}$ of beans". The authors are kindly asked to determine definitely how these concentrations are obtained? By other words what the amounts from the test material added to the substrate in each time.

Answer: Thank you for your comment. We have inserted the requested information ( $p$. 12, I. 265).

Reviewer: 11. Lines 281-283: These concentrations were based on the work of Tofel et al. (2017), who obtained LC50 of around $9 \mathrm{mg}$ of azadirachtin per $\mathrm{kg}$ of corn, using Callosobruchus maculatus (Fabricius) as the target organism. Please delete and only refer to the reference (Tofel et al. 2017).

Answer: Thank you for your comment. We have corrected this (p. 12, I. 265). Please verify the revised version of the manuscript.

Results:

Overall, this section is well written, except the fact that many parts in the beginning of each subtitle should be abbreviated.

Reviewer: 1. Lines 449-450: Consider this title as: 3.3.1. Biological activity against $A$. obtectus instead of: 3.3.1 Acanthoscelides obtectus: mortality and interaction between the nanobiopesticide and the target organism.

Answer: Thank you for your comment. We have replaced the title for "Biological activity against A. obtectus", (p. 22, I. 440). Please verify the revised version of the manuscript.

Reviewer: 2. Line 262-264: " It was also observed that the zein nanoparticles without the active compound only had an effect at the highest concentration employed, in agreement with the work of Pascoli et al. (2019)". Delete or transfer to Discussion section. The authors are kindly asked to delete any references from the Results section. Please write your own results.

Answer: Thank you for your comment. We have deleted all references from the Results section. Please verify the revised version of the manuscript. 
Reviewer: 3. Line 541-543: The effects of the formulations on the mites (larvae, nymphs, and adult females) were evaluated considering the mortality rates after direct or residual treatments. Please delete.

Answer: Thank you for your comment. We have deleted the indicated sentence.

Discussion:

Reviewer: This section is too long.

The authors are kindly asked to discuss their own results. In many parts of discussion, the authors repeated knowledge that mentioned previously in the introduction. Please abbreviate this section.

Answer: Thank you for your comment. We have abbreviated the discussion. Please verify the revised version of the manuscript.

References:

Reviewer: About 100 references are too much. Please delete the unimportant ones. Answer: Thank you for your comment. We have deleted the unimportant references, remaining 47 now. 


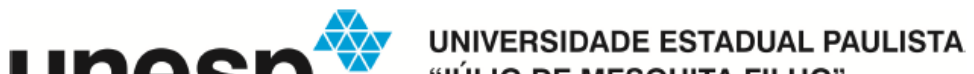 \\ "JÚLIO DE MESQUITA FILHO" \\ Instituto de Ciência e Tecnologia \\ Câmpus de Sorocaba
}

Sorocaba, August $14^{\text {th }}, 2019$

\author{
Dear Dr. Michael Traugott \\ Editor-in-Chief \\ Journal of Pest Science
}

Please find enclosed our manuscript entitled "Nanobiopesticide based on zein nanoparticles and neem oil: a study using target and nontarget organisms" from Pascoli et al. to be considered for publication as original paper in Journal of Pest Science. In this manuscript, we had developed neem oil-loaded zein nanoparticles based on an eco-friendly preparation method of encapsulation of botanical compounds aiming sustainable agriculture applications. Also, as the strategy safer by design, we investigated the phytotoxic effects on nontarget organisms (Phaseolus vulgaris) in order to correlate the potential environmental toxicity of this system with the chemical composition of the nanoparticles as well as the biological activity against worldwide pests (Acanthoscelides obtectus, Bemisia tabaci, and Tetranychus urticae). The results showed that this new carrier systems do not provoke phytotoxic effects to Phaseolus vulgaris being able to increase insecticidal effects against store pest Acanthoscelides obtectus and control of Bemisia tabaci and Tetranychus urticae. The formulations presented an attractive potential for use in crop protection in sustainable agriculture contributing to the goal of sustainability as well as increase the food security and in this way, being from interest of Journal of Pest Science readers.

Sincerely yours,

Dr. Leonardo Fernandes Fraceto

State University of São Paulo - Unesp/Sorocaba

Alto da Boa Vista, Sorocaba, São Paulo, 18087-180, Brazil, e-mail: leonardo.fraceto@unesp.br 
4 Bruno Tinoco-Nunes ${ }^{4}$; Wanderson Henrique Cruz Oliveira ${ }^{4}$; Kelly Cristina

5 Gonçalves ${ }^{5}$; Ricardo Antônio Polanczyk ${ }^{5}$; Jaqueline Franciosi Della Vechia5;

6 Sidnéia Terezinha Soares de Matos $^{5}$; Daniel Júnior de Andrade ${ }^{5}$; Halley Caixeta

7 Oliveira ${ }^{3}$; Jayme A. Souza-Neto ${ }^{4}$; Renata de Lima ${ }^{6}$; Leonardo Fernandes

8 Fraceto $^{1}$

\section{The potential of nanobiopesticide based on zein nanoparticles and neem oil for enhanced control of agricultural pests}

Mônica Pascoli ${ }^{1}$ Felícia Pereira de Albuquerque ${ }^{2}$; Anderson Kikuchi Calzavara ${ }^{3}$;

Fraceto 1

'São Paulo State University (UNESP), Institute of Science and Technology of Sorocaba, Laboratory of Environmental Nanotechnology, Av. 3 de março, 511, Alto da Boa Vista, Sorocaba, 18087-180, São Paulo, Brazil

IInstitute of Science and Technology of Sorocaba, São Paulo State University, Sorocaba, São Paulo, Brazil

3Department of Animal and Plant Biology, Londrina State University, Londrina, Paraná, Brazil

${ }^{4}$ São Paulo State University (UNESP), School of Agricultural Sciences, Department of Bioprocesses and Biotechnology, Central Multiuser Laboratory, Botucatu, Brazil

${ }^{5}$ Department of Crop Protection, College of Agricultural and Veterinary Sciences, São Paulo State University, Jaboticabal, São Paulo, Brazil ${ }^{6}$ Laboratory of Bioactivity Assessment and Toxicology of Nanomaterials, University of Sorocaba, Sorocaba, São Paulo, Brazil

Corresponding author: L. F. Fraceto, leonardo.fraceto@unesp.br +55 (15) 3238-3409 
Nanomaterials composed of natural matrices associated with biopesticides have promising applications in sustainable agriculture. In this study, the biopesticide neem oil was encapsulated in zein nanoparticles in order to improve its stability and efficiency. Assays of phytotoxicity (using Phaseolus vulgaris) and biological activity against three pests (Acanthoscelides obtectus, Bemisia tabaci, and Tetranychus urticae) were also performed. The neem oil-loaded zein nanoparticles presented $198 \pm 16 \mathrm{~nm}$, polydispersity index of around 0.2 , satisfactory physicochemical stability, together with high encapsulation efficiency exhibited mortality effects on $B$. tabaci and $T$. urticae, while the effect against $A$. in sustainable agriculture after the required toxicological assessments.

41 Keywords: Nano-scale, sustainable development, azadirachtin, phytotoxicity, biological activity, pest control. 


\section{Key Message}

- To maximize pest control and overcome adverse effects caused by synthetic pesticides, the utilization of nanobiopesticides is recommended in sustainable agriculture.

- A nanobiopesticide based on zein nanoparticles and neem oil is stable over 90 days of storage.

- Nanoencapsulation potentiated the insecticidal effects of neem oil against Acanthoscelides obtectus.

- Nanoencapsulated neem oil was effective against Bemisia tabaci and Tetranychus urticae.

- This new system showed no phytotoxicity to Phaseolus vulgaris.

- The nanobiopesticide has potential for enhanced control of agricultural pests.

\section{Author contributions}

MP and LFF designed research. MP produced and characterized the nanobiopesticide. MP, FPA, AKC, KCG, JFDV and STSM conduced biological assays. BTN, WHCO, RL, LFF and JASN contributed in analyzes of interactions between nanoparticles and organisms. MP, FPA and DJA analyzed data. LFF and $R L$ supervised the research. MP, RL and LFF wrote the manuscript. FPA, HCO, DJA, RAP, JASN, RL and LFF revised the manuscript. All authors read and approved the manuscript. 


\section{Introduction}

Crops are attacked by about 67,000 species of organisms, including

69 insects and mites, which are estimated to cause production losses ranging from 10 to $16 \%$ (Ross and Lembi 1985). In order to reduce these losses, new systems have been developed using nanotechnology to protect crops from pests such as weeds, insects, fungi, and mites, as well as to detect and treat plant diseases, deliver fertilizers and other active agents, increase nutrient and water absorption, and allow genetic exploration and transformation (Koul 2019).

Nanobiopesticides are nanomaterials with pesticidal activity or nanostructured carriers loaded with active biological compounds. Such formulations can provide greater protection of an active agent, with improved stability, absorptive capacity, and effectiveness against the target organism, while minimizing adverse effects (Borgatta et al. 2018; Oliveira et al. 2019).

These new systems should be extensively evaluated in terms of their possible risks to public health and the environment, especially where there is direct interaction between food products and nanomaterials (Pascoli et al. 2018; Kah et al. 2019; Lowry et al. 2019; Prajitha et al. 2019).

Hasheminejad et al. (2019) produced chitosan nanoparticles loaded with clove oil, which prolonged the release of the active agent and increased its antifungal activity against Aspergillus niger (van Tieghem). Campos et al. (2018a) encapsulated carvacrol and linalool in $\beta$-cyclodextrin/chitosan nanoparticles, which led to higher insecticidal activity against Helicoverpa armigera (Hübner) 
(corn earworm) and Tetranychus urticae (Koch) (two-spotted spider mite), together with lower cytotoxicity in 3T3 fibroblasts and V79 lung cells.

Oliveira et al. (2018a, 2019) used zein to encapsulate combinations of geraniol and R-citronellal, as well as geraniol, eugenol, and cinnamaldehyde. In the first study, encapsulation increased the biological activity of the compounds against $T$. urticae. In the second study, enhanced effects were observed against the same pest and Chrysodeixis includens (Walker). In both cases, there were decreased toxic effects towards nontarget organisms. Kamaraj et al. (2018) demonstrated potential antifeedant activity of neem gum-loaded nanoparticles against $H$. armigera and Spodoptera litura (Fabricius) larvae and pupae, while this nanoformulation did not affect the nontarget organism Eudrilus eugeniae (Kinberg).

Adopting the same approach, Pascoli et al. (2019) prepared neem oilloaded zein nanoparticles with a mean diameter of $278 \pm 6.1 \mathrm{~nm}$, which were stable under the experimental conditions. In vitro ecotoxicological assays showed that the new system decreased or eliminated the toxic effects of the active compound against nontarget organisms such as Allium cepa L. and Caenorhabditis elegans. In addition, the formulation did not affect soil bacteria involved in the nitrogen cycle. However, there have not yet been any tests of the biological activity of this nanoformulation towards target insects, or its potential phytotoxicity under realistic in vivo conditions.

The aim of the present study was to investigate the biological efficacy of neem oil-loaded zein nanoparticles against three species of agricultural pest: i) the bean beetle Acanthoscelides obtectus (Say), ii) the whitefly Bemisia tabaci 
113 (Gennadius), and iii) the two-spotted spider mite T. urticae as target organisms.

114 The phytotoxic effects of these nanoparticles against Phaseolus vulgaris L. was also evaluated. The stability of the nanoparticles was investigated during 90 days, using measurements of mean hydrodynamic diameter, polydispersity index, span index, zeta potential, nanoparticle concentration, and encapsulation efficiency. This innovative study opens perspectives for the use of nanobiopesticides based on neem and zein nanoparticles in pest control.

\section{Materials and Methods}

\subsection{Supplies}

Zein (catalogue number P1300, 88 - 96\% purity) and Pluronic F-68 (catalogue number 9010-66-6) were obtained from Sigma-Aldrich. Neem oil (Azamax) was acquired from UPL Brazil. Absolute Ethanol (code AE07218RA, 99.5\%) was purchased from Labsynth. The 18:1 Liss Rhod PE fluorophore (1,2dipalmitoyl-sn-glycero-3-phosphoethanolamine-N-(lissamine rhodamine B sulfonyl) (ammonium salt)), code 810158, was acquired from Avanti Polar Lipids. Seeds of common bean ( $P$. vulgaris cultivar IPR Curió, Carioca group, register 30616, protection 20130167) were kindly supplied by the Agronomic Institute of Paraná (IAPAR, Londrina, Parana, Brazil). Stored grain beetles (A. obtectus) were obtained from a colony maintained at the Biology Laboratory of São Paulo State University (UNESP, Sorocaba, São Paulo, Brazil). Whitefly (B. tabaci) and two-spotted spider mite ( $T$. urticae) were obtained from colonies maintained at São Paulo State University (UNESP, Jaboticabal, São Paulo, Brazil). Other chemicals, reagents, and solvents used were purchased from local suppliers. 


\subsection{Preparation of neem oil-loaded zein nanoparticles}

Zein nanoparticles were prepared by the anti-solvent precipitation method, described by $\mathrm{Hu}$ and McClements (2014), after treatment of zein as performed by Pascoli et al. (2019). Firstly, zein (2\% w/v) was solubilized in a hydroethanolic solution $(85 \% \mathrm{v} / \mathrm{v})$, under magnetic stirring overnight. The $\mathrm{pH}$ of the zein solution was adjusted to 5.8 , followed by centrifugation for $30 \mathrm{~min}$ at $85750 \mathrm{xg}$, heat treatment at $75{ }^{\circ} \mathrm{C}$ for $15 \mathrm{~min}$, and filtering through a $0.45 \mu \mathrm{m}$ membrane (Millipore). A $100 \mathrm{mg}$ aliquot of neem oil (containing $12 \mathrm{~g} / \mathrm{L}$ of azadirachtin) was added to the zein solution. An aqueous solution of Pluronic F-68 (2\% v/v) was prepared and the $\mathrm{pH}$ was adjusted to 4 . The zein solution containing neem oil was rapidly injected into the Pluronic solution, under magnetic stirring. The colloidal formulation was stirred at room temperature, in order to evaporate the ethanol, and water $(\mathrm{pH} 4.0)$ was added to complete to $20 \mathrm{~mL}$. The final concentration of neem oil in the nanoformulation was $5 \mathrm{mg} / \mathrm{mL}$. In field, neem oil is used at concentrations between 4 and $6 \mathrm{mg} / \mathrm{mL}$, thus, an intermediate concentration was chosen for the formulation. Control nanoparticles were prepared without neem oil. Labeled nanoparticles, with and without neem oil, were also prepared with addition of rhodamine (18:1 Liss Rhod PE) in the zein solution $(0.05 \% \mathrm{~m} / \mathrm{m}$, relative to the polymer), in order to investigate the interaction between the seed beetles and the formulation (Gott et al. 2014).

\subsection{Physico-chemical stability of the nanoparticles}


159 Determinations of the mean hydrodynamic diameter and the polydispersity index 160 of the nanoparticles were performed by photon correlation spectroscopy, using a 161 ZetaSizer Nano ZS 90 analyzer (Malvern Instruments) at a fixed angle of $90^{\circ}$ and temperature of $25^{\circ} \mathrm{C}$. The same equipment was used to determine the zeta potential, according to the microelectrophoresis method (Grillo et al. 2012). The mean nanoparticle diameter was also determined using NanoSight Nanoparticle LM10 instrument (Malvern Panalytical) and the span index (an indicator of the stability of the formulation, showing the width of the size distribution), was calculated as follows:

$$
\text { Span }=\frac{(D 90-D 10)}{D 50}
$$

where D10, D50, and D90 are the mean diameters corresponding to 10, 50, and $90 \%$ of the particle population, respectively. The particle concentrations in the formulations were also measured using a NanoSight equipped with a $532 \mathrm{~nm}$ laser. The images were collected using a sCMOS camera and were processed using NanoSight v. 2.3 software (Grillo et al. 2014). For these analyses, the samples were diluted 1000 times. The efficiency of encapsulation of the neem oil in the zein nanoparticles was quantified using the ultrafiltration/centrifugation method, with analysis using a UV-Vis spectrophotometer (Cary 50, Varian). The samples were centrifuged using Microcon $10 \mathrm{kDa}$ regenerated cellulose ultrafilters (Millipore), which only allowed passage of the unencapsulated neem. The analytical curve concentration range was from 10 to $200 \mu \mathrm{g} / \mathrm{mL}$ and detection employed a wavelength of $225 \mathrm{~nm}$ (Dubhashi et al. 2013). The encapsulation efficiency was calculated by the difference between the amount of neem initially added and the filtered amount obtained. 


\subsection{Phytotoxicity evaluation using bean plants}

The substrate used for plant growth was clay soil and sand, in a ratio of 1:1 (v:v). The pots and growing trays were kept in the greenhouse of the Center of Biological Sciences of Londrina State University (Londrina, Paraná, Brazil), under natural conditions of air relative humidity and temperature, with $75 \%$ of total environmental photosynthetic photon flux density (PPFD). The soil was enriched with the nutrient solution of Hoagland and Arnon (1950) and was regularly watered. Pre- and post-emergence assays were performed, with the following treatments: water (negative control), zein nanoparticles, neem oil-loaded zein nanoparticles, and neem oil. The concentration adopted in each application of these treatments was the same as that recommended for the commercial product: $5 \mathrm{mg} / \mathrm{mL}$ applied at 100 liters per hectare.

For the post-emergence assay, three applications to the leaves of bean plants were performed, with intervals of 7 days. Each treatment was applied to seven pots, each with three seedlings. At the beginning of the experiment, only the first pair of leaves was fully expanded; hence, all the chlorophyll a fluorescence, gas exchange, and oxidative stress analyses were performed using these leaves. In the pre-emergence assay, the treatments were applied once, directly to the soil of five pots (each with 25 seeds), using amounts equivalent to the three applications of the post-emergence test.

Chlorophyll a fluorescence was measured at the adaxial surfaces of the leaves, using an OS1p fluorometer (Opti-Sciences, Hudson, USA). The maximum quantum yield of photosystem II photochemistry $\left(F_{v} / F_{m}\right)$ was determined as follows: 
208 where $F_{0}$ refers to the minimum, $F_{m}$ to the maximum, and $F_{v}$ to the variable 209 fluorescence of dark-adapted leaves after receiving a saturating pulse of actinic light (Baker, 2008). Gas exchange analyses were performed to determine the light-saturated net photosynthesis $\left(A_{\max }\right)$, using a portable infrared gas analyzer (Model $6400 \mathrm{XT}$, LI-COR Biosciences, Lincoln, USA) connected to a $6 \mathrm{~cm}^{2}$ chamber. The saturating PPFD inside the chamber during the analyses was $1,500 \mu \mathrm{mol} \mathrm{m} \mathrm{m}^{-2} \mathrm{~s}^{-1}$, as determined previously using a light-curve analysis. In the post-emergence assay, the analyses were always carried out two days after application of the treatments to the plants, at the same times (07:30 a.m. for $F_{v} / F_{m}$ and 08:30 a.m. for $\left.A_{\max }\right)$. In the pre-emergence assay, the analyses were performed only at the end of the experiment, at the same time-points described.

Hydrogen peroxide and lipid peroxidation were measured as markers of oxidative stress. For these analyses, $100 \mathrm{mg}$ portions of fresh leaves and roots were ground to a powder in liquid nitrogen, followed by extraction with $1.8 \mathrm{~mL}$ of methanol $+0.2 \%$ trichloroacetic acid (TCA). After centrifugation (13700 xg for 5 $\min$ at $4^{\circ} \mathrm{C}$ ), the supernatant was used for measurement of the hydrogen peroxide content by reaction with potassium iodide, in phosphate buffer (Alexieva et al. 2001), and for the determination of thiobarbituric acid reactive substances (TBARS) (Camejo et al. 1998). For determination of hydrogen peroxide, the supernatant was subjected to reaction for one hour with $1 \mathrm{M}$ potassium iodide (KI), in $\mathrm{pH} 7.5$ phosphate buffer (PBS), keeping the mixture on ice and in the dark. at $390 \mathrm{~nm}$, using a 96-well plate and a microplate reader (Model Victor TM 3, 
231 PerkinEImer, Turku, Finland). For determination of TBARS, the supernatant was

232 subjected to reaction with $0.02 \%$ butylated hydroxytoluene $(\mathrm{BHT})$ in $\mathrm{pH} 7.4 \mathrm{PBS}$

233 buffer, together with $1.3 \%$ thiobarbituric acid (TBA) and $0.3 \%$ sodium hydroxide

$234(\mathrm{NaOH})$, in the presence of $50 \% \mathrm{TCA}$, at $60{ }^{\circ} \mathrm{C}$ for $60 \mathrm{~min}$. Lipid peroxidation

235 concentration was determined using a malondialdehyde (MDA) standard curve

236 constructed from fluorescence readings obtained at excitation and emission

237 wavelengths of 535 and $590 \mathrm{~nm}$, respectively, employing the Victor TM 3 reader

238 (Camejo et al. 1998).

239 Finally, for plant dry mass determination, the plants were harvested (after

24013 days in the pre-emergence assay and after 24 days in the post-emergence 241 assay), individually packed in paper bags, and dried in an oven at $60{ }^{\circ} \mathrm{C}$ until 242 reaching constant mass.

\section{$243 \quad 2.5$ Biological activity assays}

\subsubsection{Evaluation of mortality of Acanthoscelides obtectus and its} interaction with the nanobiopesticide

The bioassays using $A$. obtectus were conducted in the Biology Laboratory of UNESP/ICTS, in controlled climate chambers with constant aeration, absence of light, temperature of $27 \pm 2{ }^{\circ} \mathrm{C}$, and maximum and minimum humidity of 73 and $52 \%$, respectively, based on the studies of Jumbo et al. (2014), Soares et al. (2014), and Janković-Tomanić et al. (2015). The colony was maintained under the same conditions. The Phaseolus vulgaris (Qualitá ${ }^{\circledR}$ ) used to maintain the culture and to carry out the experiments was previously kept in a freezer for 14 days and dried, in order to prevent possible infestation from the field and to 
254 reduce any potential effect of insecticide residue, as proposed by Jumbo et al. 255 (2014).

The biocidal activity assays were carried out according to the method described by Jumbo et al. (2014), using an acute mortality assay (96 h) to estimate the mean lethal concentration $\left(\mathrm{LC}_{50}\right)$. Masses of $25 \mathrm{~g}$ of beans were placed in $145 \mathrm{~mL}$ plastic bottles with small holes in the cap for aeration, followed by application of the treatments (zein nanoparticles, neem oil-loaded zein nanoparticles and neem oil) and shaking the vials manually for 60 seconds to ensure complete distribution of the material in the beans. Ten unsexed adults (1 to 5 day old) of $A$. obtectus were placed in each vial. The experiment was carried out with concentrations equivalent to $1.35,2.7,5.4,10.8$, and $21.6 \mathrm{mg}$ of azadirachtin per $\mathrm{kg}$ of beans (Tofel et al. 2017), using 0.5, 1.12, 2.25, 4.5 and 9 $\mathrm{mL}$ of formulation, respectively. After the exposure period, mortality was evaluated using a stereomicroscope (Model XTB-2B, Coleman), with the beetles being considered dead when they did not show movement, even when stimulated by touching with a fine-bristle brush for $4 \mathrm{~min}$. Two replicates were performed for each dose and for the control treatment, and the experiment was repeated three times. The LC50 values were estimated as proposed by Hamilton et al. (1977), using the Trimmed Spearman-Karber method.

The treatments with the rhodamine-labeled nanoparticles were performed in the same way, under the same experimental conditions as described for the $A$. obtectus biological activity assay, using the $\mathrm{LC}_{50}$ concentration for the neem oilloaded nanoparticles and the same volume for the zein nanoparticles without the active agent. The insetcs were analyzed at the Central Multiusers Laboratory of the School of Agricultural Sciences (UNESP) after $96 \mathrm{~h}$ of exposure, using a Carl 
279 Zeiss SteREO Discovery v. 12 microscope fitted with a red filter for fluorescence,

280 in order to identify the presence of the nanoformulation in the bodies of the 281 insects. The images were acquired with an Axiocam 2.0 Zen Blue camera and 282 were treated using the equipment software. The images of the bodies of $A$. obtectus were merged with the fluorescence evaluation images, enabling visualization of the interactions between the beetles and the treatments. A total of 10 specimens were analyzed for each treatment. Untreated control specimens were used to evaluate any possible natural fluorescence emitted by the body of the insect.

\subsubsection{Bemisia tabaci mortality assay}

The whitefly (B. tabaci) mortality experiments were conducted in the Microbial Control of Pest Arthropods Laboratory (UNESP/FCAV). The whiteflies used in this assay were reared on bean plants in a greenhouse and were collected in flat bottom glass tubes, using manual suction. A total of 480 insects were collected in 48 tubes (10 insects per tube). These tubes were transferred to the previously treated bean plants in pots (24 pots, each with 2 plants) and were left open until the flies had emerged from the tubes. Prior to the transfer of the whiteflies, the treatments were applied to the bean plants by manual spraying, as recommended by the manufacturer of the commercial neem oil (3 applications, spaced at intervals of 7 days). Three scenarios with different concentrations were simulated: concentration of $5 \mathrm{mg} / \mathrm{mL}, 100 \mathrm{~L} /$ hectare (also as recommended by the manufacturer), concentration estimating overdosage $(15 \mathrm{mg} / \mathrm{mL}, 100$ 
$\mathrm{mg} / \mathrm{mL}, 100 \mathrm{~L} /$ hectare). Six replicates were performed for each treatment and the dead insects found on the floors of the cages were counted daily.

\subsubsection{Biological effects on Tetranychus urticae}

The assays of biological effects against the T. urticae mite were conducted in the Acarology Laboratory (UNESP/FCAV), using mites obtained from jack bean plants (Canavalia ensiformes L.). The plants were cultivated in $2 \mathrm{~L}$ pots containing soil, sand, and bovine manure (1:1:1, v:v:v) as the substrate. The mites were kept in a temperature-controlled climate chamber at $25 \pm 1 \stackrel{\circ}{ } \mathrm{C}$, relative humidity (RH) of $60 \pm 10 \%$, and $12 \mathrm{~h} / 12 \mathrm{~h}$ light/dark photoperiod. The experiments were performed using arenas ( $2.5 \mathrm{~cm}$ diameter) of $C$. ensiformes leaves obtained using a circular metal cutter. The arenas were placed in Petri dishes $(9 \times 2 \mathrm{~cm})$ containing a moistened foam and a hydrophilic cotton layer $(1.0 \mathrm{~cm})$, in order to maintain the turgidity of the arenas, and were surrounded with hydrophilic cotton to avoid escape of the mites.

Evaluations of biological activity were performed using the larvae, nymphs, and adults of $T$. urticae. The treatments (water as the negative control, zein nanoparticles, zein nanoparticles with neem oil at $5 \mathrm{mg} / \mathrm{mL}$, neem oil at $5 \mathrm{mg} / \mathrm{mL}$, and the commercial synthetic acaricide Oberon $^{\circledR}$ as a positive control) were evaluated for direct and residual action. For evaluation of the direct action, the mites in the different stages of development (larvae, nymphs, or adult females) were transferred to the arenas (10 mites per arena). The treatments were then

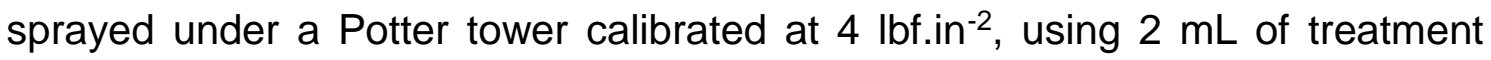
solution per arena, corresponding to $1.56 \mathrm{mg} . \mathrm{cm}^{-2}$ of dry residue. Each treatment 
was repeated 8 times. After the applications, the arenas were transferred to a climate-controlled chamber, as described above. For the residual evaluation of the formulations, jack bean (C. ensiformes) seeds were planted in $5 \mathrm{~L}$ pots containing soil, sand, and bovine manure (1:1:1, v:v:v) as substrate. Approximately 30 days after germination, the plants were separated into 5 groups of three plants to receive the applications of the different treatments. The products were applied with a $500 \mathrm{~mL}$ capacity manual sprayer, until complete coverage of the plants. An average of $15 \mathrm{~mL}$ of treatment solution was required per plant. After 1,6 , and 12 days following the applications, leaves of the bean plants were collected and arenas were prepared in Petri dishes, as described above, followed by the transfer of 10 larvae, nymphs, or adults to each arena. Each assay employed 8 replicates. The numbers of mites that were alive, dead, or trapped in the cotton barrier were counted daily during 5 days, using a stereomicroscope (40x magnification). Mites that did not react to the touch of a fine brush were considered dead.

\subsection{Statistical analysis}

The results of the biological activity assays were treated as proposed by Abbott (1925) for corrected mortality. The statistical analyses were performed with GraphPad Prism v. 6 software, using one-way ANOVA for stability, two-way ANOVA for phytotoxicity and biological activity assays against Acanthoscelides obtectus and Tetranychus urticae, and repeated measures ANOVA for Bemisia tabaci mortality followed by the Tukey post-hoc test, at a significance level of $p<0.05$. 


\section{Results}

\subsection{Physico-chemical stability of the nanoparticles}

In this study, the physico-chemical stability of the neem oil-loaded zein

351

352

353

354

355

356

357

358

359

360

361

362

363

364

365

366

367

368

369

nanoparticles was evaluated by determination of several parameters during storage of the formulations for 90 days. Initially, mean diameter (Figure 1A) was obtained by DLS (288 $\pm 6 \mathrm{~nm})$ and it showed a significant increase on day 60 reaching an average diameter of $313 \pm 8.1 \mathrm{~nm}(F=15.54, D F=5, P<0.0001)$. Using the same technique, the polydispersity index (Figure 1A) was found to remain at around 0.2 , with a decrease on day $10(F=7.387, D F=5, P=0.0022)$. and no other significant differences between day 10 and 90, indicating good physicochemical stability of the polymer system. Use of the NTA technique, which enables determination of the hydrodynamic diameter of the particles by directly measuring their diffusion coefficients when they are in Brownian motion, resulted in nanobiopesticide particle sizes that were smaller than obtained by DLS, with $198 \pm 16 \mathrm{~nm}$ (Figure 1B). Using this technique, the mean diameters oscillated significantly, increasing on day 20 and 60 and decreasing on day 10 and 90 ( $F=$ 59.17, $D F=5, P<0.0001)$ throughout the storage time, which could have been because the technique is more sensitive and analyzes each particle individually. The span index values (Figure 1B) were less than 1 and showed significant decrease only on day $10(F=7.387, D F=5, P=0.0022)$. No other significant differences during the 90 days of storage were observed, which is also a characteristic of stable formulations. 
371 significant fluctuations during the 90 days of storage $(F=172.5, D F=5, P<$ $0.0001)$ nanoparticles (Figure 1D) showed that the highest encapsulation efficiency of 86 $\pm 0.5 \%$ was obtained on day 5 , followed by a significant gradual decrease to 64 $\pm 0.6 \%$ after 90 days $(F=588.6, D F=5, P<0.0001)$, which remained constant

377 until day 90 . The release of the active agent from the nanoparticles over time could be responsible for this decrease in encapsulation efficiency.

a)

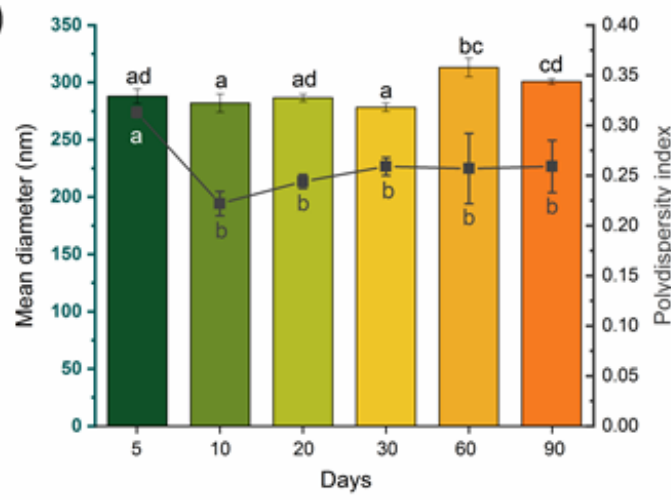

c)

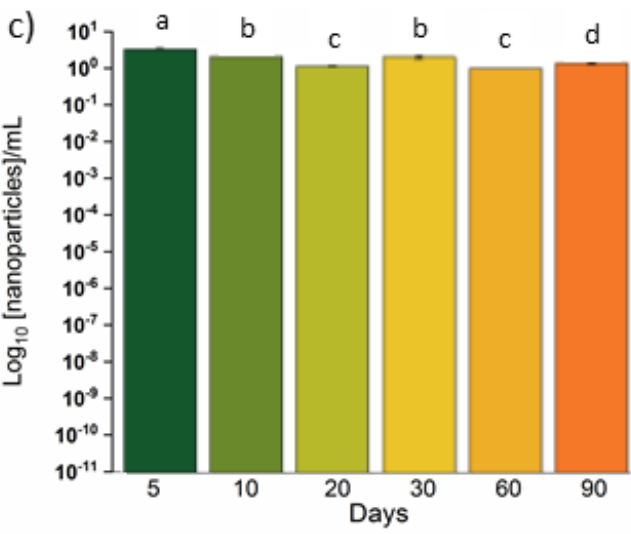

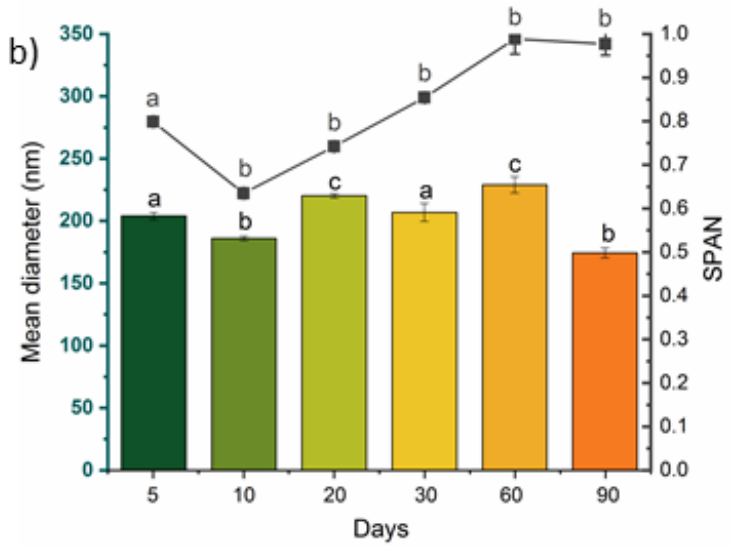

d)

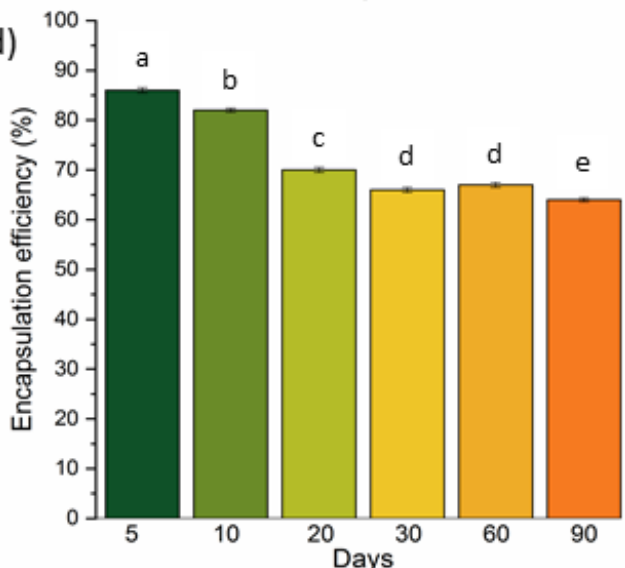

Fig. 1 Stability of the neem oil-loaded zein nanoparticles during 90 days: A) Mean hydrodynamic size (bars) and polydispersity index (line), obtained using DLS. B) Mean hydrodynamic size (bars) and span index (line), obtained using NTA. C) 
383 Concentration of nanoparticles in the formulation, obtained by NTA. D)

384 Encapsulation efficiency of neem oil in the zein nanoparticles, obtained by UV-

385 Vis spectroscopy. The data are expressed as the average of three independent experiments $(n=3)$ and the error bars represent the standard deviations. Equal letters indicate values that do not differ significantly according to one-way ANOVA followed by the Tukey post-hoc test $(p<0.05)$.

The zeta potential values (data not shown) oscillated significantly during the 90 days of storage $-36 \pm 1 \mathrm{mV}$ on day $1,-41 \pm 2.9 \mathrm{mV}$ on day $10,-24.6 \pm 0.8$ $\mathrm{mV}$ on day 20 and $-15.5 \pm 2.5 \mathrm{mV}$ on day $60(F=86.41, D F=5, P<0.0001)$ indicating a lack of stability. However, Pluronic F-68 was used during the nanoparticles preparation process, which provided steric hindrance and was responsible for the stability of the system.

\subsection{Phytotoxicity evaluation using bean plants}

The $F_{v} / F_{m}$ ratio, which indicates the maximum quantum efficiency of electron transport in photosystem II, was not affected by any of the formulations tested, regardless of the type of treatment (Table 1). All the leaves presented $F_{v} / F_{m}$ values near 0.8 . The $A_{\max }$ values for the treated plants showed no significant differences, compared to the corresponding controls, evidencing that 402 the formulations did not affect photosynthetic activity in the leaves. In the third evaluation of the plants in the post-emergence test, there was a significant decrease of $A_{\max }$, relative to the first and second evaluation of the same plants ( $F$ $405=938.6, D F=2, P<0.0001)$. However, this result, verified in all treatments 
406 (including the control), is justifiable by the senescence presented by the leaf used 407 for the analyses.

Similar to the photosynthetic parameters, lipid peroxidation and hydrogen peroxide levels in the roots and leaves showed no significant differences between

410 the control and the treatments (Table 1), demonstrating that the formulations did not induce oxidative stress in common bean plants.

413 Table 1 Maximum quantum yield of photosystem II photochemistry $\left(F_{v} / F_{m}\right)$, lightsaturated net photosynthesis ( $\left.A_{\max }\right)$, and oxidative stress parameters of the bean plants. $1^{\text {st }}, 2^{\text {nd }}$, and $3^{\text {rd }}$ represent the analyses after the first, second, and third treatment applications, respectively. The data are expressed as average \pm 417 standard deviation for three $(n=3)$ analyses using ten (10) and fourteen (14) plants for the pre- and post-emergence assays, respectively. The symbols $\dagger$ and $\phi$ indicate significant difference relative to the $1^{\text {st }}$ and $2^{\text {nd }}$ analyses, respectively, according to two-way ANOVA followed by the Tukey post-hoc test $(p<0.05)$.

\begin{tabular}{|c|c|c|c|c|c|c|}
\hline \multirow[b]{2}{*}{ Treatments } & \multirow[t]{2}{*}{$F_{v} / F_{m}$} & \multirow[t]{2}{*}{$\begin{array}{c}A_{\max }\left(\mu \mathrm{mol} \mathrm{CO}_{2}\right. \\
\left.\mathrm{m}^{-2} \mathrm{~s}^{-1}\right)\end{array}$} & \multicolumn{2}{|c|}{ 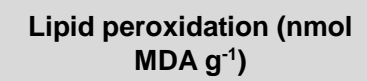 } & \multicolumn{2}{|c|}{$\mathrm{H}_{2} \mathrm{O}_{2}\left(\mu \mathrm{mol} \mathrm{g}{ }^{-1}\right)$} \\
\hline & & & root & leaf & root & leaf \\
\hline \multicolumn{7}{|c|}{ Pre-emergence assay } \\
\hline Control & $0.774 \pm 0.011$ & $15.8 \pm 3.3$ & $12.7 \pm 2.8$ & $29.2 \pm 5.8$ & $31.0 \pm 2.3$ & $332.2 \pm 12.3$ \\
\hline Zein NP & $0.760 \pm 0.021$ & $16.5 \pm 2.0$ & $9.6 \pm 3.2$ & $36.6 \pm 3.0$ & $35.3 \pm 3.4$ & $356.1 \pm 19.5$ \\
\hline Neem NP & $0.753 \pm 0.015$ & $17.5 \pm 2.3$ & $7.2 \pm 4.4$ & $32.8 \pm 9.1$ & $25.3 \pm 4.8$ & $334.9 \pm 40.4$ \\
\hline Neem & $0.767 \pm 0.019$ & $16.2 \pm 2.0$ & $12.6 \pm 8.8$ & $33.8 \pm 5.9$ & $27.9 \pm 4.2$ & $356.7 \pm 33.0$ \\
\hline \multicolumn{7}{|c|}{ Post-emergence assay } \\
\hline $1^{\text {st }}$ Control & $0.826 \pm 0.008$ & $25.9 \pm 3.1$ & - & - & - & - \\
\hline $1^{\text {st }}$ Zein NP & $0.827 \pm 0.007$ & $25.6 \pm 3.0$ & - & - & - & - \\
\hline $1^{\text {st }}$ Neem NP & $0.829 \pm 0.006$ & $23.5 \pm 1.6$ & - & - & - & - \\
\hline
\end{tabular}




\begin{tabular}{l|l|l|c|c|c|c}
$\mathbf{1}^{\text {st }}$ Neem & $0.830 \pm 0.005$ & $26.5 \pm 2.5$ & - & - & - & - \\
$\mathbf{2}^{\text {nd }}$ Control & $0.794 \pm 0.015$ & $16.7 \pm 2.4$ & - & - & - & - \\
$\mathbf{2}^{\text {nd }}$ Zein NP & $0.792 \pm 0.019$ & $17.3 \pm 1.1$ & - & - & - & - \\
$\mathbf{2}^{\text {nd }}$ Neem NP & $0.788 \pm 0.008$ & $17.1 \pm 2.2$ & - & - & - & - \\
$\mathbf{2}^{\text {nd }}$ Neem & $0.791 \pm 0.020$ & $16.75 \pm 1.7$ & - & - & - & $362.7 \pm 39.8$ \\
$\mathbf{3}^{\text {rd }}$ Control & $0.790 \pm 0.017$ & $4.1 \pm 2.1 \dagger^{\phi}$ & $12.7 \pm 4.6$ & $47.5 \pm 5.8$ & $28.2 \pm 16.2$ & \\
$\mathbf{3}^{\text {rd }}$ Zein NP & $0.785 \pm 0.019$ & $5.2 \pm 2.8 \dagger^{\phi}$ & $12.3 \pm 5.5$ & $48.3 \pm 4.8$ & $18.3 \pm 12.5$ & $373.9 .1 \pm 40.2$ \\
$\mathbf{3}^{\text {rd }}$ Neem NP & $0.808 \pm 0.005$ & $6.6 \pm 3.1 \dagger^{\phi}$ & $14.2 \pm 4.3$ & $50.1 \pm 3.0$ & $25.60 \pm 20.5$ & $450.9 \pm 48.9$ \\
$\mathbf{3}^{\text {rd }}$ Neem & $0.797 \pm 0.014$ & $5.8 \pm 2.8 \dagger^{\phi}$ & $7.8 \pm 3.4$ & $51.0 \pm 5.5$ & $12.3 \pm 10.3$ & $422.8 \pm 44.4$
\end{tabular}

423 analyses, the dry mass of the bean plants did not show any significant difference 424 among the control and the treatments in the pre- and post-emergence 425 experiments. This demonstrates that the biopesticide and the neem oil did not 426 affect the growth of the plants under the experimental conditions adopted (Figure 427 2). 


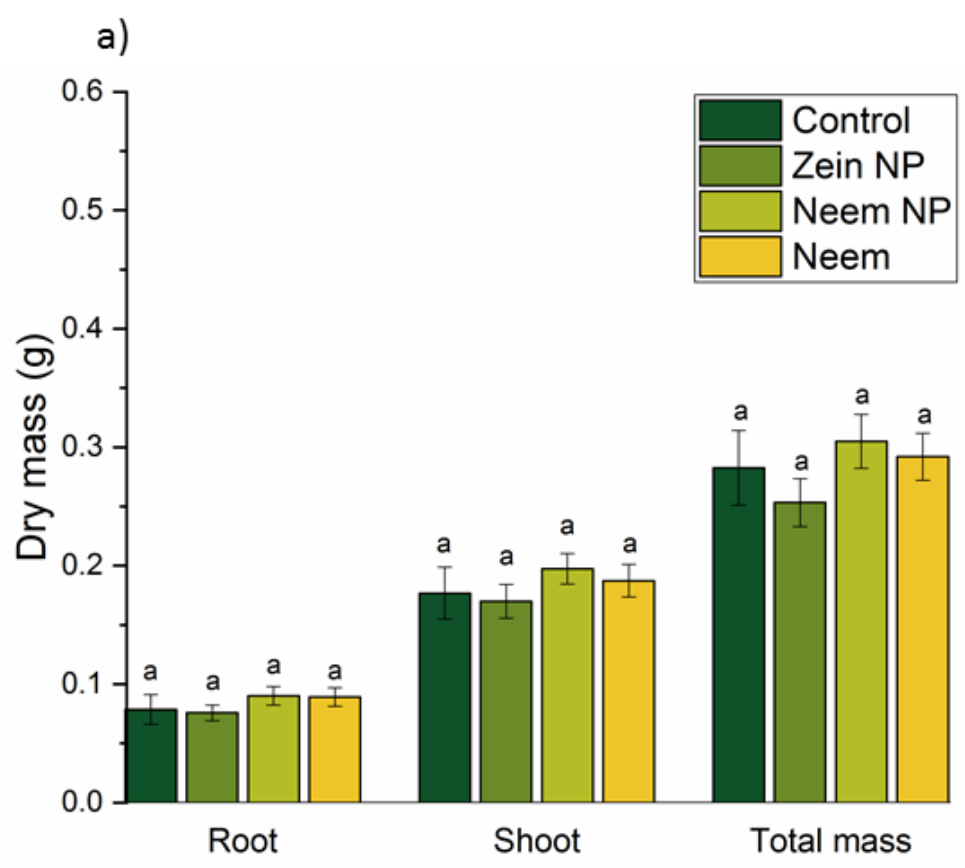

b)

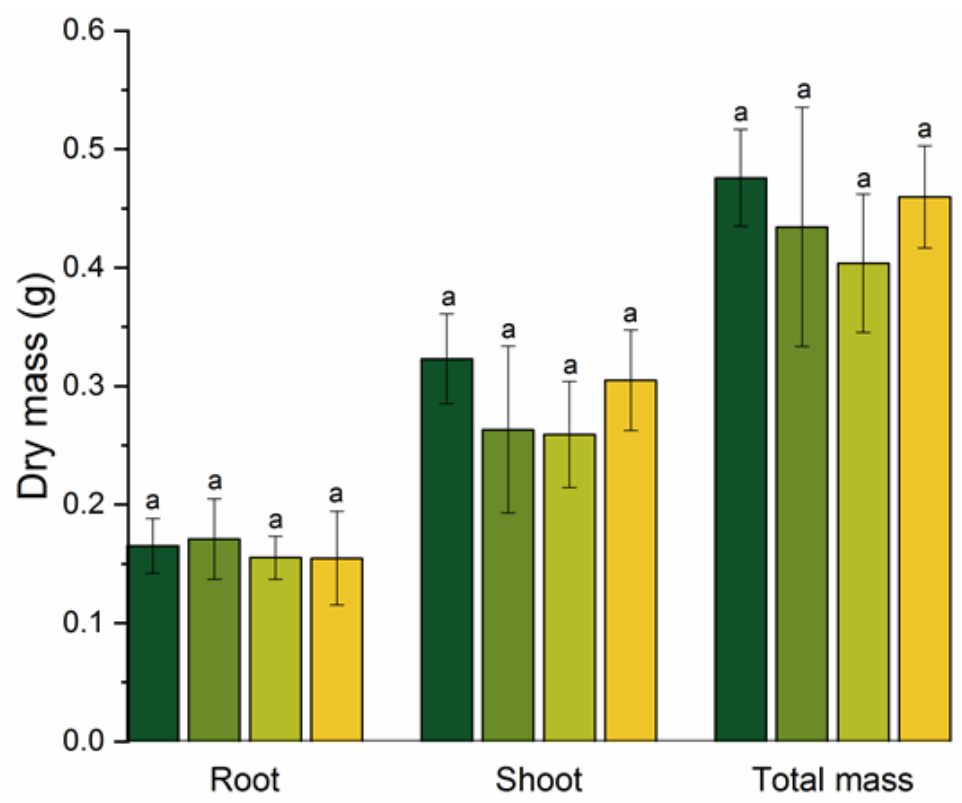

Fig. 2 Results of phytotoxicity assays using common bean plants: Dry masses of 431 plants treated with water (control), zein nanoparticles (Zein NP), neem oil-loaded 432 zein nanoparticles (Neem NP), and neem oil (Neem). A) Pre-emergence assay; 433 B) post-emergence assay. The data are expressed as averages of ten $(n=10)$ 434 and fourteen $(n=14)$ plants for the pre- and post-emergence assays, 435 respectively. The error bars represent the standard deviations. Equal letters 
436 indicate values that do not differ significantly according to one-way ANOVA

437 followed by the Tukey post-hoc test $(p<0.05)$.

\subsection{Biological activity}

\subsubsection{Biological activity against Acanthoscelides obtectus} Spearman-Karber method, according to the confidence interval of the results. The LC50 values were $6.65 \mathrm{mg}$ of azadirachtin per $\mathrm{kg}$ of beans for the neem oilloaded zein nanoparticles and $11.22 \mathrm{mg}$ of azadirachtin per $\mathrm{kg}$ of beans for the neem oil, indicating that the new system provided greater efficiency against this bean pest, compared to the traditional neem oil.

The results (Figure $3 \mathrm{~A}$ ) showed that the neem oil nanobiopesticide caused significant mortality of the pest from the second lowest concentration tested, while the neem oil only caused significant mortality at the highest concentration evaluated. It was also observed that the zein nanoparticles without the active compound only had an effect at the highest concentration employed $(F=24.00$, $D F=3, P<0.0001)$

In order to evaluate the contact between the nanobiopesticide and the 454 insects, the nanobiopesticide was labeled with the 18:1 Liss Rhod PE 455 fluorophore. The resulting material had the same physical chemical characteristics as the unlabeled nanobiopesticide (data not shown). 
458 exposure of the $A$. obtectus individuals to the nanoformulations was mainly via 459 the integument (Figure 3B), with the greatest exposure occurring in the ventral 460 region, especially the legs and mouthparts. Nanoparticles could also be seen on 461 the antennae and the abdomen. These results suggested that the increased 462 mortality of $A$. obtectus (Figure 3A) was probably due to direct contact and 463 interaction with the nanobiopesticide, with better adhesion facilitating absorption 464 of the nanostructures by the insect. 


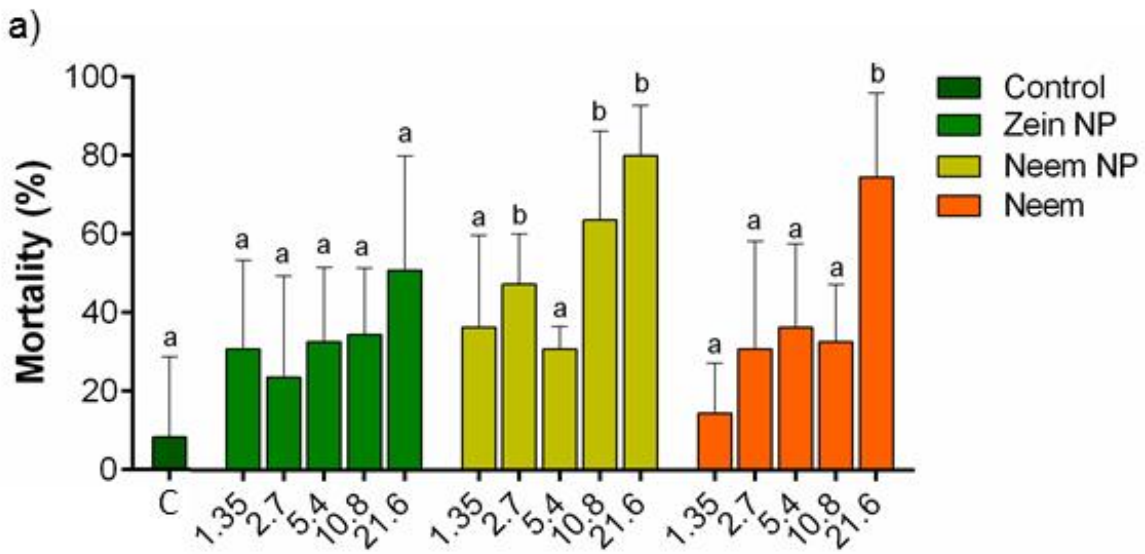

b)

Concentration ( $\mathrm{mg}$ azadirachtin/ $\mathrm{kg}$ bean)
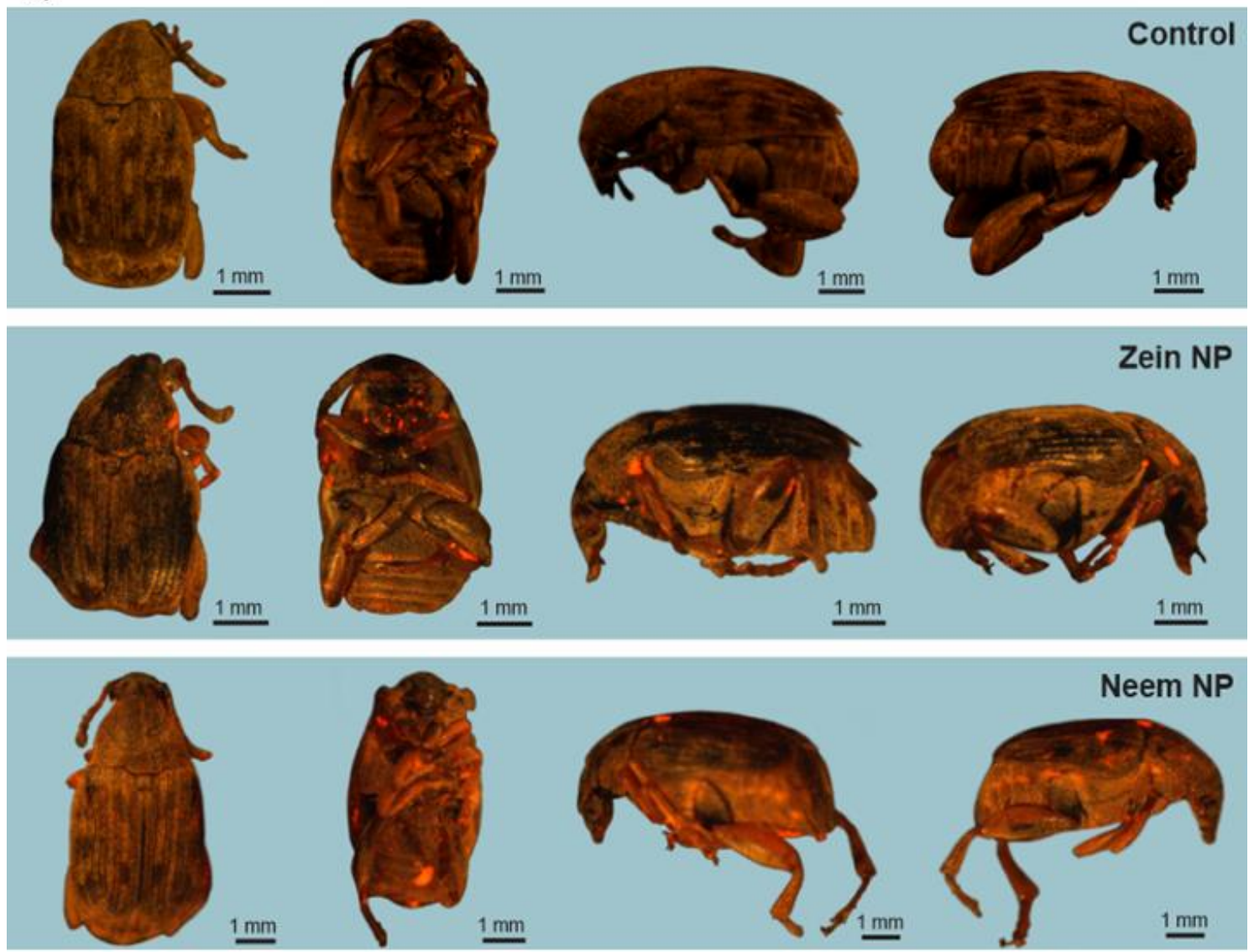

467 Fig. 3 Results of assays using Acanthoscelides obtectus: A) Mortality of $A$. 468 obtectus following acute exposure $(96 \mathrm{~h})$ to beans treated with the zein 469 nanoparticles (Zein NP), the neem oil-loaded zein nanoparticles (Neem NP), and 470 the neem oil (Neem), at concentrations of $1.35,2.7,5.4,10.8$, and $21.6 \mathrm{mg}$ of 
471 azadirachtin per $\mathrm{kg}$ of beans. The zein nanoparticle treatment was used as a

472 control, at the same volume as the treatments containing the active agent. B)

473 Images of $A$. obtectus exposed for $96 \mathrm{~h}$ to beans treated with neem oil-loaded

474 zein nanoparticles labeled with rhodamine (Neem NP), at a concentration of 6.64

$475 \mathrm{mg}$ of azadirachtin per $\mathrm{kg}$ of beans. Labeled zein nanoparticles and untreated

476 bruchines were used as a control. The data are expressed as the average of

477 three independent experiments $(n=3)$, normalized to \%. The error bars represent

478 the standard deviation. Equal letters indicate values that do not differ significantly

479 according to two-way ANOVA followed by the Tukey post-hoc test $(p<0.05)$.

\subsubsection{Biological effect on Bemisia tabaci}

Figure 4 shows the results of the mortality assays using the nanoformulations and neem oil against $B$. tabaci. The treatments were performed at concentrations of $5 \mathrm{mg} / \mathrm{mL}$, as recommended by the manufacturer of commercial neem oil, $15 \mathrm{mg} / \mathrm{mL}$, representing overdosage, and $1 \mathrm{mg} / \mathrm{mL}$, representing less use of the bioinsecticide.

In the assay performed under the use conditions recommended by the manufacturer (Figure 4A), the mortality of the pest presented significant increases, compared to the control, starting on the $3^{\text {rd }}$ day for the neem oil, and on the $5^{\text {th }}$ day for the zein nanoparticles with neem oil. In this case, the commercial neem oil showed no higher efficiency than the neem oil-loaded zein nanoparticles $(F=7.22, D F=18, P<0.0001)$. 

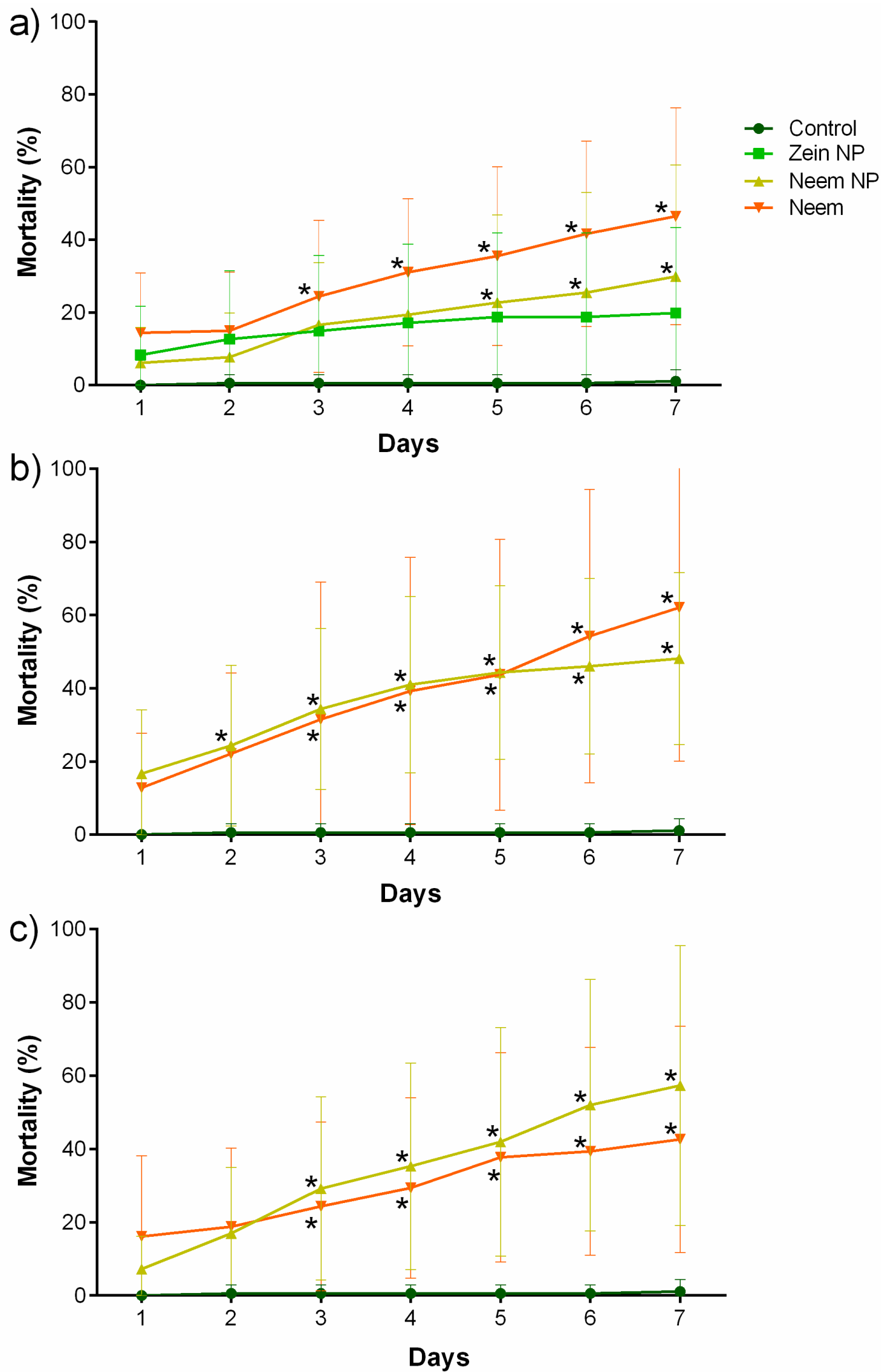

494

Fig. 4 Mortality of whiteflies treated with zein nanoparticles (Zein NP), neem oil496 loaded zein nanoparticles (Neem NP), and neem oil (Neem), at A) the 
497 recommended concentration (5 mg/mL), B) overdosage concentration (15 $498 \mathrm{mg} / \mathrm{mL})$, and C) lower dosage $(1 \mathrm{mg} / \mathrm{mL})$. The data are expressed as averages 499 of three independent experiments $(n=3)$, normalized to \%. The error bars 500 represent the standard deviation. The symbol * indicate significant difference 501 relative to control. A significance level of $P<0.05$ was adopted using repeated 502 measures ANOVA followed by the Tukey post-hoc test.

503

In the overdosage scenario (Figure 4B), the treatments presented significantly higher mortality compared to the control from day 2 to day $7(F=$ 10.46, $D F=12, P<0.0001)$, with no significant difference between the treatments. Considering the capacity of $B$. tabaci to develop resistance to pesticides, the increase in mortality could be attributed to the increase of the concentration of the applied active compound.

In the assay using lower concentrations of the bioinsecticide (Figure 4C), the mortality results were again similar for the neem oil and the neem oil-loaded nanoparticles, and significantly higher compared to the control from day $3(F=$ 16.65, $D F=12, P<0.0001)$. However, calculation of the areas under the curves (Table 2) revealed that in the experiment carried out using the neem oil at a concentration of $1 \mathrm{mg} / \mathrm{mL}$, the nanobiocide and the neem oil showed the same result with areas of 207.7 and 179 , respectively, showing the potential for using a lower concentration of the pesticide to control whitefly.

519 Table 2 Area under the curve values for the biological activity assays using the 520 control and the nanobiopesticide at concentrations of 5,15 , and $1 \mathrm{mg} / \mathrm{mL}$ : water 
521 (Control), zein nanoparticles (Zein NP), neem-loaded zein nanoparticles (Neem

$522 \mathrm{NP}$ ), and neem oil (Neem). The data are expressed as the average \pm standard

523 deviation of three independent experiments $(n=3)$. Different letters denote

524 significant differences. A significance level of $P<0.05$ was adopted using two-

525 way ANOVA followed by the Tukey post-hoc test.

\begin{tabular}{|c|c|}
\hline Treatments & $\begin{array}{c}\text { Area under the curve } \\
\text { (mortality } \mathbf{x} \text { days) }\end{array}$ \\
\hline \multicolumn{2}{|c|}{$\mathbf{5} \mathbf{~ m g / m L}$ assay } \\
\hline Control & $3.33 \pm 4.06^{\mathrm{a}}$ \\
Zein NP & $96.37 \pm 36.72^{\mathrm{b}}$ \\
\hline Neem NP & $110.00 \pm 36.61^{\mathrm{b}}$ \\
\hline Neem & $178.10 \pm 38.39^{\mathrm{b}}$ \\
\hline \multicolumn{2}{|c|}{$\mathbf{1 5} \mathbf{~ m g / m L}$ assay } \\
\hline Neem NP & $222.40 \pm 39.45^{\mathrm{b}}$ \\
\hline Neem & $228.10 \pm 60.02^{\mathrm{b}}$ \\
\hline \multicolumn{2}{|c|}{$\mathbf{1} \mathbf{~ m g / m L}$ assay } \\
\hline Neem NP & $207.70 \pm 48.28^{\mathrm{b}}$ \\
Neem & $179.00 \pm 44.36^{\mathrm{b}}$ \\
\hline
\end{tabular}

526

527

\subsubsection{Tetranychus urticae mortality}

528

Figure 5 shows the mortality rates following direct application of the treatments (at a neem oil concentration of $5 \mathrm{mg} / \mathrm{mL}$ ) to the larvae (Figure $5 \mathrm{~A}$ ), nymphs (Figure 5B), and adults (Figure 5C). For the larvae and nymphs, use of

531 the neem oil-loaded nanoparticles led to a slightly higher mortality rate, compared 532 to use of the neem oil, although the differences were not significant. However, 533 both neem oil and the neem oil-loaded zein nanoparticles showed acaricide potential against $T$. urticae, exceeding $50 \%$ mortality, with a similar result for the positive control $(F=1.09, D F=3, P=0.3684$ and $F=3.08, D F=3, P=0.0436$, 
536 respectively). It was interesting to note that the zein nanoparticles caused mortality of the mites, especially when applied to the larvae, where the mortality rates were similar to those observed for the insecticide.

540 (Figure 5D), nymphs (Figure 5E), and adults (Figure 5F), with the mortality rates generally decreasing over time $(F=23.06, D F=11, P<0.0001, F=66.34, D F=$ 11, $P<0.0001$ and $F=38.41, D F=11, P<0.0001$, respectively). The most efficient results were observed on the first day after application $(F=31.33, D F=$ 3, $P<0.0001, F=19.67, D F=3, P<0.0001$ and $F=11.68, D F=3, P<0.0001$, respectively), which were comparable to the results obtained in the direct treatment (Figures $5 \mathrm{~A}, 5 \mathrm{~B}$, and $5 \mathrm{C}$ ). A possible explanation for this was that in the case of the residual treatment (Figures 5D, 5E, and 5F), the leaves were attached to the plants at the time of application, so the active metabolism could have led to the treatments reaching the leaves, resulting in the mites ingesting more of the active ingredient. However, over time, the compounds were degraded and their efficiencies decreased.

An exception to the reduction in mortality over time in the residual effect assays was observed for the effect of the neem nanoparticles on the larvae (Figure 5D), where larval mortality increased on the $12^{\text {th }}$ day. This could be attributed to the ability of the nanoparticles to protect the active agent, hence prolonging its effectiveness, under the experimental conditions employed. 

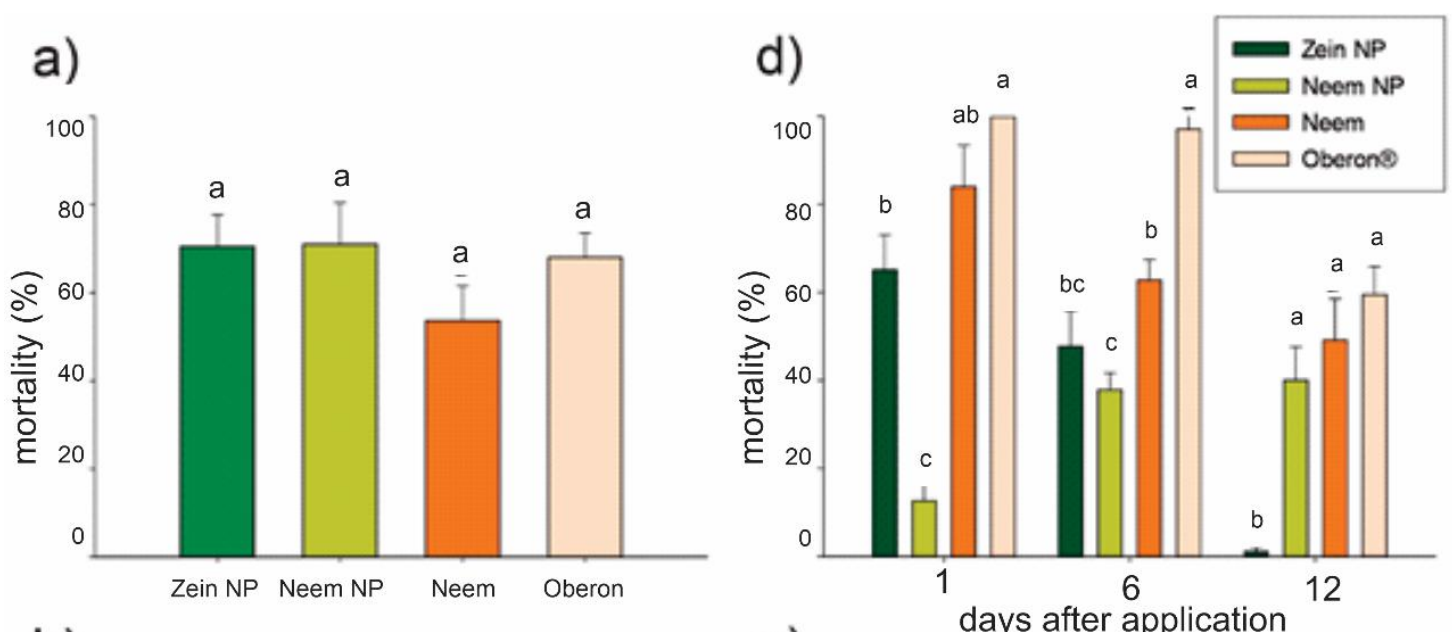

b)
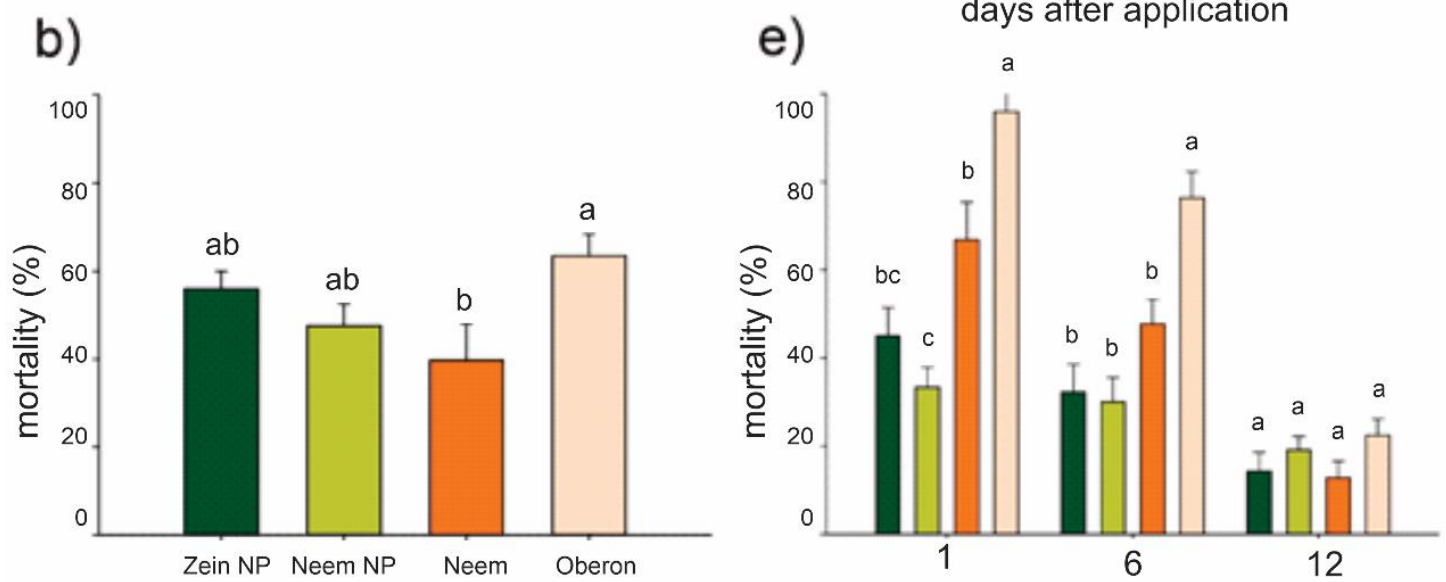

c)
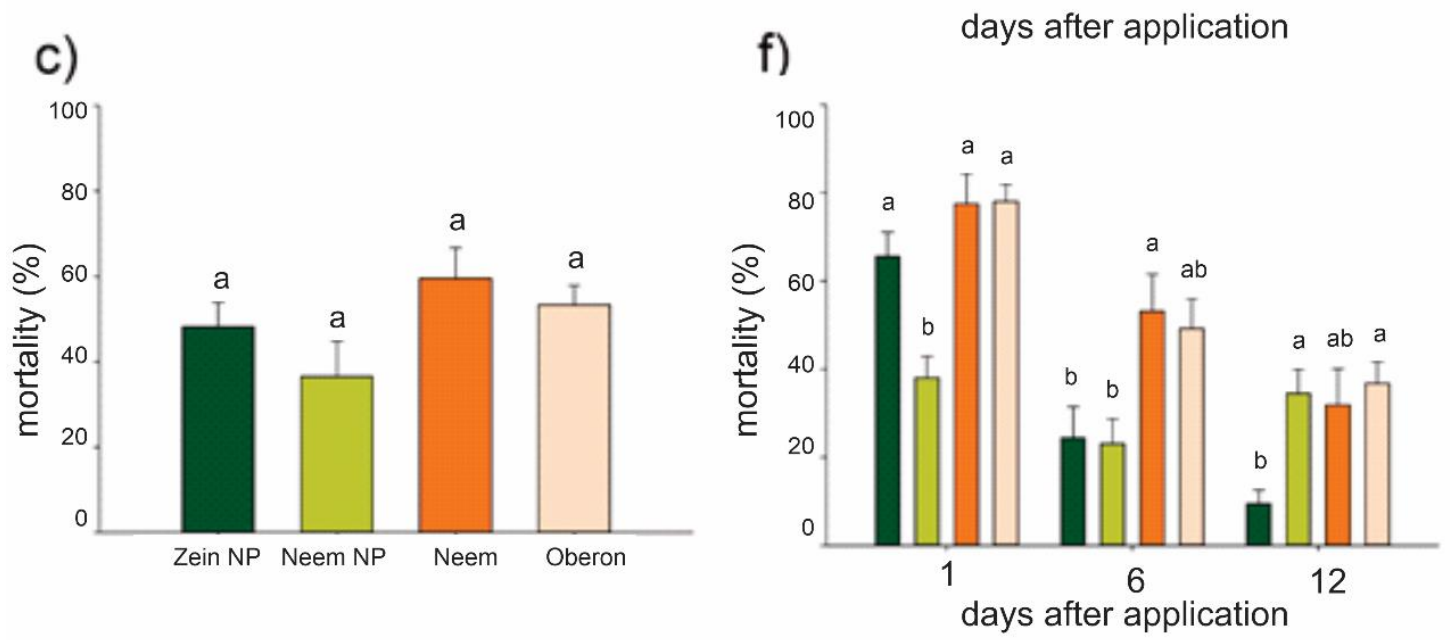

559 Fig. 5 Results of biological activity assays using Tetranychus urticae. Mortality 5

560 days after direct applications on the A) larvae, B) nymphs, and C) adults, using

561 zein nanoparticles (Zein NP), neem oil-loaded zein nanoparticles (Neem NP),

562 neem oil (Neem), and Oberon ${ }^{\circledR}$ (acaricide as positive control). Residual effects

563 on the D) larvae, E) nymphs, and F) adults analyzed on leaves collected 1, 2 and

5646 days after the application of the treatments. The data are expressed as the 
565 averages of eight repetitions $(n=8)$, normalized to \%. The error bars represent

566 the standard deviation. Different letters denote significant differences. A 567 significance level of $P<0.05$ was adopted using two-way ANOVA followed by the 568 Tukey post-hoc test.

\section{Discussion}

In relationship to the nanoparticle's characterization, the mean diameter obtained by DLS was higher than that obtained using NTA. A similar result was reported by Oliveira et al. (2018a) for zein nanoparticles. The encapsulation efficiency shows that novel zein nanocarrier systems have promising potential for the encapsulation and protection of active compounds. The negative potential zeta results were in agreement with the findings of Podaralla and Perumal (2012) and Oliveira et al. (2019), who used Pluronic F-68 to obtain zein nanoparticles

The physico-chemical stability results showed that although the nanoparticles in suspension presented oscillations of the mean diameter, the polydispersion and span indices remained similar to the values characteristic of stable formulations. The nanoparticle concentration also showed no significant alterations, while the encapsulation efficiency decreased, as expected since the nanocarrier released the active compound as a function of time. Nonetheless, despite the release, the loading still remained at $70 \%$, which could be considered high. Therefore, it could be concluded that the presence of Pluronic F-68 as a surfactant was effective in maintaining the stability of the nanobiopesticide.

Given that pest control would lead to plants being exposed to high concentrations of nanoformulations, the phytotoxicity of new nanotechnological 
systems should be carefully investigated (Yu et al. 2015). The photosynthetic activity and the growth of $P$. vulgaris plants were not affected by the nanoformulation, as well as it did not induce oxidative stress in plant cells. Taken together, these results indicated that this new nanobiopesticide is safe for application to $P$. vulgaris under the experimental conditions adopted. Our results corroborate the reports by Sridharan et al. (2015) and Oliveira et al. (2018a), which showed that neem oil and zein nanoparticles did not demonstrate phytotoxic potential, emerging as a tool for pest control in sustainable agriculture.

In contrast, this new nanobiopesticide increased insecticidal effects against store pest $A$. obtectus, which is one of the most important pests of $P$. vulgaris dry beans, multiplying in the field and post-harvest (Vuts et al. 2018). This insect has a wide variety of host plants and reduces the mass, volume, physiological quality, and germination index of beans, while increasing the temperature and water content, leading to losses of around 7-40\% (Mbogo et al. 2009). Bean producers and distributors control $A$. obtectus using insecticides including pyrethroids, organophosphates, and aluminum phosphide fumigant (Pimentel et al. 2012). However, the use of these compounds has led to concerns regarding environmental contamination, pest resistance evolution, and impacts on human health (Shelef et al. 2018; Pellegrini and Fernández 2018). Hence, this new technology for the control of $A$. obtectus that can contribute to safety in agriculture.

Also, the findings with the images of $A$. obtectus exposed to neem oilloaded zein nanoparticles labeled with rhodamine which show the nanobiopesticide in the ventral region, mouthpart and antennae open perspectives for improving understanding of the effects of nanoformulations. 
Using B. tabaci, another most serious polyphagous pests of field and

614 greenhouse crops, was observed the potential for using a lower concentration of

615 the neem oil to control whitefly. Different to the assay performed with $A$. obtectus

616 (which showed a directly proportional relationship between concentration

617 increase and insecticidal effect), a possible explanation for this result was that at

618 the lower concentration, the nanoparticles presented greater dispersion, which

619 reduced the possibility of aggregation and enhanced the capacity of the

620 nanoparticles to enter into contact with the organism, even penetrating its

621 integument.

An important point was that although the neem oil commercial product was

recommended for use against this pest, the mortality shown was lower than expected (not reaching 50\%), which could have been due to the great ability of

B. tabaci to develop resistance to pesticides. In addition, the different populations

of $B$. tabaci present genetic differences that could be responsible for important biological differences among them, in terms of symbionts, feeding behavior, virus transmission, host plant variety, and resistance to insecticides (Harish et al. 2019; Hussain et al. 2019; Wang et al. 2019).

According to these results, Kumar (2008) reported mortality in B. tabaci using commercial neem oil (NeemAzal-U 17\%) under semi-field conditions and Boursier et al. (2011) found that neem plant extract had the same effect on whitefly as commercial neem oil. Campos et al. (2018a) and Oliveira et al. (2019) studied the effects of polymeric nanoparticle formulations containing essential oils against $H$. armigera and $C$. includens, respectively, and in both cases, a greater sublethal effect was obtained using the encapsulated compounds, compared to commercial compounds. On the other hand, Oliveira et al. (2018b) 
638 found that chitosan/gum arabic nanoparticles loaded with eugenol had an

639

640

641

642

643

644

645

646

647

648

649

650

651

652

653

654

655

656

657

658

659

660

661

attractive effect for $B$. tabaci. It can be seen from these results that the effect of the active agent can vary according to its form and the experimental conditions, which emphasizes the need to carry out an extensive evaluation of any new system.

Finally, in relationship to $T$. urticae, considering that it is a pest that exhibits fast reproductive capacity and resistance to a wide range of active agents, this nanobiopesticide may be promising for field application, since it can confer protection of the active agent which led to prolonged effects and consequently reduce the need for reapplication of the product on the larvae, indicating the potential benefits of these nanotechnological products in agricultural applications. In the same way, Ahmadi et al. (2018) and Campos et al. (2018a) also showed the ability of nanoencapsulation to increase the acaricidal activities of natural compounds against $T$. urticae.

In summary, the nanobiopesticide based on zein nanoparticles containing neem oil showed good physicochemical stability during 90 days. It is important to emphasize that the encapsulation of the active compound significantly increased its effectiveness against the pest $A$. obtectus and fluorescence labeling of the nanoparticles enabled visualization of the interaction of the nanomaterial with the test organism. Besides, this new system had no phytotoxic effects on common bean plants under our experimental conditions and presented biological activity against whitefly (B. tabaci) and two-spotted spider mite (T. urticae). Therefore, the present findings provide further support for the excellent potential of this nanobiopesticide to be used in pest control in sustainable agriculture. 


\section{Acknowledgments}

663 The authors are grateful for the financial support for this research provided by the

664 São Paulo Research Foundation (FAPESP, grants \#2017/21004-5, 665 \#2015/17120-4, and \#2013/11343-6) and the National Council for Scientific and 666 Technological Development (CNPq).

667 Conflicts of interest

668 There are no conflicts of interest to declare.

\section{Human and animal rights}

This article does not contain any studies with human participants or animals

671 (vertebrates) performed by any of the authors.

\section{References}

Ahmadi Z, Saber M, Akbari A, Mahdavinia GR (2018) Encapsulation of Satureja hortensis L. (Lamiaceae) in chitosan/TPP nanoparticles with enhanced acaricide activity against Tetranychus urticae Koch (Acari: Tetranychidae). Ecotoxicol Environ Saf 161:111-119. https://doi.org/10.1016/j.ecoenv.2018.05.051

Alexieva V, Sergiev I, Mapelli S, Karanov E (2001) The effect of drought and ultraviolet radiation on growth and stress markers in pea and wheat. Plant Cell Environ 24:1337-1344

Borgatta J, Ma C, Hudson-Smith N, et al (2018) Copper Based Nanomaterials Suppress Root Fungal Disease in Watermelon ( Citrullus lanatus ): Role of Particle Morphology, Composition and Dissolution Behavior. ACS Sustain Chem Eng 6:14847-14856. https://doi.org/10.1021/acssuschemeng.8b03379 
Boursier CM, Bosco D, Coulibaly A, Negre M (2011) Are traditional neem extract preparations as efficient as a commercial formulation of azadirachtin A? Crop Prot 30:318-322. https://doi.org/10.1016/j.cropro.2010.11.022

Camejo G, Wallin B, Enojärvi M (1998) Analysis of oxidation and antioxidants using microtiter plates. Free Radic Antioxid Protoc Methods Mol Biol 108:377-386

Campos EVR, Proença PLF, Oliveira JL, et al (2018) Carvacrol and linalool coloaded in $\beta$-cyclodextrin-grafted chitosan nanoparticles as sustainable biopesticide aiming pest control. Sci Rep 8:. https://doi.org/10.1038/s41598-018-26043-x

Corrêa AS, Pereira EJG, Cordeiro EMG, et al (2011) Insecticide resistance, mixture potentiation and fitness in populations of the maize weevil (Sitophilus zeamais). Crop Prot 30:1655-1666. https://doi.org/10.1016/j.cropro.2011.08.022

Fraceto LF, Grillo R, de Medeiros GA, et al (2016) Nanotechnology in Agriculture: Which Innovation Potential Does It Have? Front Environ Sci 4:. https://doi.org/10.3389/fenvs.2016.00020

Gott RC, Luo Y, Wang Q, Lamp WO (2014) Development of a biopolymer nanoparticle-based method of oral toxicity testing in aquatic invertebrates. Ecotoxicol Environ Saf 104:226-230. https://doi.org/10.1016/j.ecoenv.2014.03.017

Grillo R, Abhilash PC, Fraceto LF (2016) Nanotechnology Applied to BioEncapsulation of Pesticides. J Nanosci Nanotechnol 16:1231-1234. https://doi.org/10.1166/jnn.2016.12332

Grillo R, dos Santos NZP, Maruyama CR, et al (2012) Poly( $\varepsilon$ caprolactone)nanocapsules as carrier systems for herbicides: Physicochemical characterization and genotoxicity evaluation. J Hazard Mater 231-232:1-9. https://doi.org/10.1016/j.jhazmat.2012.06.019

Grillo R, Pereira AES, Nishisaka CS, et al (2014) Chitosan/tripolyphosphate nanoparticles loaded with paraquat herbicide: An environmentally safer alternative for weed control. J Hazard Mater 278:163-171. https://doi.org/10.1016/j.jhazmat.2014.05.079

Hamilton MA, Russo RC, Thurston RV (1977) Trimmed Spearman-Karber method for estimating median lethal concentrations in toxicity bioassays. Environ Sci Technol 11:714-719. https://doi.org/10.1021/es60130a004

Harish ER, K. M, T. M, et al (2019) Next generation sequencing reveals endosymbiont variability in cassava whitefly, Bemisia tabaci, across the agro-ecological zones of Kerala, India. Genome gen-2018-0050. https://doi.org/10.1139/gen-2018-0050 
Hasheminejad N, Khodaiyan F, Safari M (2019) Improving the antifungal activity of clove essential oil encapsulated by chitosan nanoparticles. Food Chem 275:113-122. https://doi.org/10.1016/j.foodchem.2018.09.085

Hoagland DR The water-culture method for growing plants without soil. 32

$\mathrm{Hu} \mathrm{K}$, McClements DJ (2014) Fabrication of surfactant-stabilized zein nanoparticles: A pH modulated antisolvent precipitation method. Food Res Int 64:329-335. https://doi.org/10.1016/j.foodres.2014.07.004

Hussain S, Farooq M, Malik HJ, et al (2019) Whole genome sequencing of Asia II 1 species of whitefly reveals that genes involved in virus transmission and insecticide resistance have genetic variances between Asia II 1 and MEAM1 species. BMC Genomics 20:507. https://doi.org/10.1186/s12864019-5877-9

Janković-Tomanić M, Jovanović DŠ, Savković U, et al (2015) Host expansion modifies activity of phosphatases in a legume store pest Acanthoscelides obtectus (Say). J Stored Prod Res 62:32-35. https://doi.org/10.1016/j.jspr.2015.03.008

Kamaraj C, Gandhi PR, Elango G, et al (2018) Novel and environmental friendly approach; Impact of Neem ( Azadirachta indica ) gum nano formulation (NGNF) on Helicoverpa armigera (Hub.) and Spodoptera litura (Fab.). Int J Biol Macromol 107:59-69. https://doi.org/10.1016/j.ijbiomac.2017.08.145

Koul O (2019) Nano-biopesticides today and future perspectives, 1st edition. Elsevier, Cambridge, MA

Kumar P (2008) Studies on loss of bio-efficacy of two indirect neem application over time (seed and soil) against Bemisia tabaci (Homoptera: Aleyrodidae) under semi-field conditions. J Asia-Pac Entomol 11:185-190. https://doi.org/10.1016/j.aspen.2008.08.003

Malaikozhundan B, Vaseeharan B, Vijayakumar S, Thangaraj MP (2017) Bacillus thuringiensis coated zinc oxide nanoparticle and its biopesticidal effects on the pulse beetle, Callosobruchus maculatus. J Photochem Photobiol B 174:306-314. https://doi.org/10.1016/j.jphotobiol.2017.08.014

Mbogo KP, Davis J, Myers JR (2009) Transfer of the ArcelinPhytohaemagglutinin-Ît Amylase Inhibitor Seed Protein Locus from Tepary bean (Phaseolus acutifolius A. Gray) to Common Bean (P. vulgaris L.). Biotechnology(Faisalabad) 8:285-295. https://doi.org/10.3923/biotech.2009.285.295

Mutungi C, Affognon HD, Njoroge AW, et al (2015) Triple-Layer Plastic Bags Protect Dry Common Beans ( Phaseolus vulgaris ) Against Damage by Acanthoscelides obtectus (Coleoptera: Chrysomelidae) During Storage. J Econ Entomol 108:2479-2488. https://doi.org/10.1093/jee/tov197 
Oliveira JL de, Campos EVR, Pereira AES, et al (2018a) Zein Nanoparticles as Eco-Friendly Carrier Systems for Botanical Repellents Aiming Sustainable Agriculture. J Agric Food Chem 66:1330-1340. https://doi.org/10.1021/acs.jafc.7b05552

Oliveira JL, Campos EVR, Germano-Costa T, et al (2019) Association of zein nanoparticles with botanical compounds for effective pest control systems. Pest Manag Sci 75:1855-1865. https://doi.org/10.1002/ps.5338

Oliveira JL, Campos EVR, Pereira AES, et al (2018b) Geraniol Encapsulated in Chitosan/Gum Arabic Nanoparticles: A Promising System for Pest Management in Sustainable Agriculture. J Agric Food Chem 66:53255334. https://doi.org/10.1021/acs.jafc.8b00331

Oliveira JL, Ramos Campos EV, Fraceto LF (2018c) Recent Developments and Challenges for Nanoscale Formulation of Botanical Pesticides for Use in Sustainable Agriculture. J Agric Food Chem 66:8898-8913. https://doi.org/10.1021/acs.jafc.8b03183

Pascoli M, Jacques MT, Agarrayua DA, et al (2019) Neem oil based nanopesticide as an environmentally-friendly formulation for applications in sustainable agriculture: An ecotoxicological perspective. Sci Total Environ 677:57-67. https://doi.org/10.1016/j.scitotenv.2019.04.345

Pascoli M, Lopes-Oliveira PJ, Fraceto LF, et al (2018) State of the art of polymeric nanoparticles as carrier systems with agricultural applications: a minireview. Energy Ecol Environ 3:137-148. https://doi.org/10.1007/s40974-018-0090-2

Pellegrini P, Fernández RJ (2018) Crop intensification, land use, and on-farm energy-use efficiency during the worldwide spread of the green revolution. Proc Natl Acad Sci 115:2335-2340. https://doi.org/10.1073/pnas.1717072115

Pimentel MAG, Faroni LRA, Corrêa AS, Guedes RNC (2012) Phosphine-induced walking response of the lesser grain borer (Rhyzopertha dominica). Pest Manag Sci 68:1368-1373. https://doi.org/10.1002/ps.3314

Podaralla S, Perumal O (2012) Influence of Formulation Factors on the Preparation of Zein Nanoparticles. AAPS PharmSciTech 13:919-927. https://doi.org/10.1208/s12249-012-9816-1

Prajitha N, Athira SS, Mohanan PV (2019) Bio-interactions and risks of engineered nanoparticles. Environ Res 172:98-108. https://doi.org/10.1016/j.envres.2019.02.003

Prasad R, Kumar V, Prasad SK (2014) Nanotechnology in sustainable agriculture: Present concerns and future aspects. Afr J Biotechnol 13:705713. https://doi.org/10.5897/AJBX2013.13554

Ross MA, Lembi CA (1985) Applied weed science. Burgess Pub. Co, Minneapolis, Minn. ISMB 978-0-8087-2958-7. 
Shelef O, Fernández-Bayo JD, Sher Y, et al (2018) Elucidating Local Food Production to Identify Principles and Challenges for Sustainable Agriculture. In: Sustainable Food Systems from Agriculture to Industry. Charis Galanakis, pp 47-81

Soares MA, Quintela ED, Mascarin GM, Arthurs SP (2014) Effect of temperature on the development and feeding behavior of Acanthoscelides obtectus (Chrysomelidae: Bruchinae) on dry bean (Phaseolus vulgaris L.). J Stored Prod Res 61:90-96. https://doi.org/10.1016/j.jspr.2014.12.005

Sridharan S, Shekhar KC, Ramakrishnan N (2015) Bioefficacy, Phytotoxicity, and Biosafety of Mineral Oil on Management of Whitefly in Okra. Int J Veg Sci 21:28-35. doi: 10.1080/19315260.2013.818607

Tofel KH, Kosma P, Stähler M, et al (2017) Insecticidal products from Azadirachta indica and Plectranthus glandulosus growing in Cameroon for the protection of stored cowpea and maize against their major insect pests. Ind Crops Prod 110:58-64. https://doi.org/10.1016/j.indcrop.2017.09.051

Verma ML (2017) Nanoscience in food and agriculture. 4: ... Springer, Cham

Viteri Jumbo LO, Faroni LRA, Oliveira EE, et al (2014) Potential use of clove and cinnamon essential oils to control the bean weevil, Acanthoscelides obtectus Say, in small storage units. Ind Crops Prod 56:27-34. https://doi.org/10.1016/j.indcrop.2014.02.038

Vuts J, Woodcock CM, König L, et al (2018) Host shift induces changes in mate choice of the seed predator Acanthoscelides obtectus via altered chemical signalling. PLOS ONE 13:e0206144. https://doi.org/10.1371/journal.pone.0206144

Yu Z, Sun X, Song H, et al (2015) Glutathione-Responsive Carboxymethyl Chitosan Nanoparticles for Controlled Release of Herbicides. MAS 06:591-604. doi: 10.4236/msa.2015.66062

Wang H-L, Lei T, Xia W-Q, et al (2019) Insight into the microbial world of Bemisia tabaci cryptic species complex and its relationships with its host. Sci Rep 9:6568. https://doi.org/10.1038/s41598-019-42793-8 
3 Mônica Pascoli ${ }^{1}$; Felícia Pereira de Albuquerque ${ }^{2}$; Anderson Kikuchi Calzavara ${ }^{3}$;

4 Bruno Tinoco-Nunes ${ }^{4}$; Wanderson Henrique Cruz Oliveira ${ }^{4}$; Kelly Cristina

5 Gonçalves ${ }^{5}$; Ricardo Antônio Polanczyk ${ }^{5}$; Jaqueline Franciosi Della Vechia5;

6 Sidnéia Terezinha Soares de Matos ${ }^{5}$; Daniel Júnior de Andrade ${ }^{5}$; Halley Caixeta

7 Oliveira ${ }^{3}$; Jayme A. Souza-Neto4; Renata de Lima6; Leonardo Fernandes

8 Fraceto $^{1}$

${ }^{1}$ São Paulo State University (UNESP), Institute of Science and Technology of

10 Sorocaba, Laboratory of Environmental Nanotechnology, Av. 3 de março, 511,

11 Alto da Boa Vista, Sorocaba, 18087-180, São Paulo, Brazil

12 Institute of Science and Technology of Sorocaba, São Paulo State University,

13 Sorocaba, São Paulo, Brazil

3Department of Animal and Plant Biology, Londrina State University, Londrina,

Paraná, Brazil

${ }^{4}$ São Paulo State University (UNESP), School of Agricultural Sciences, Department of Bioprocesses and Biotechnology, Central Multiuser Laboratory, Botucatu, Brazil

5Department of Crop Protection, College of Agricultural and Veterinary Sciences, São Paulo State University, Jaboticabal, São Paulo, Brazil

${ }^{6}$ Laboratory of Bioactivity Assessment and Toxicology of Nanomaterials, University of Sorocaba, Sorocaba, São Paulo, Brazil

Corresponding author: L. F. Fraceto, leonardo.fraceto@unesp.br +55 (15) 3238-3409 
Nanomaterials composed of natural matrices associated with biopesticides have promising applications in sustainable agriculture. In this study, the biopesticide neem oil was encapsulated in zein nanoparticles in order to improve its stability and efficiency. Assays of phytotoxicity (using Phaseolus vulgaris) and biological activity against three pests (Acanthoscelides obtectus, Bemisia tabaci, and Tetranychus urticae) were also performed. The neem oil-loaded zein nanoparticles presented $198 \pm 16 \mathrm{~nm}$, polydispersity index of around 0.2 , satisfactory physicochemical stability, together with high encapsulation efficiency cause any phytotoxic effects towards $P$. vulgaris. The neem oil nanobiopesticide exhibited mortality effects on $B$. tabaci and $T$. urticae, while the effect against $A$. obtectus was significantly increased, compared to plain neem oil. The results of the characterization, toxicity, and biological activity studies showed the promising potential of these neem oil-loaded zein nanoparticles for use in pest management in sustainable agriculture after the required toxicological assessments.

41 Keywords: Nano-scale, sustainable development, azadirachtin, phytotoxicity, biological activity, pest control. 


\section{Key Message}

- To maximize pest control and overcome adverse effects caused by synthetic pesticides, the utilization of nanobiopesticides is recommended in sustainable agriculture.

- A nanobiopesticide based on zein nanoparticles and neem oil is stable over 90 days of storage.

- Nanoencapsulation potentiated the insecticidal effects of neem oil against Acanthoscelides obtectus.

- Nanoencapsulated neem oil was effective against Bemisia tabaci and Tetranychus urticae.

- This new system showed no phytotoxicity to Phaseolus vulgaris.

- The nanobiopesticide has potential for enhanced control of agricultural pests.

\section{Author contributions}

MP and LFF designed research. MP produced and characterized the nanobiopesticide. MP, FPA, AKC, KCG, JFDV and STSM conduced biological assays. BTN, WHCO, RL, LFF and JASN contributed in analyzes of interactions between nanoparticles and organisms. MP, FPA and DJA analyzed data. LFF and $R L$ supervised the research. MP, RL and LFF wrote the manuscript. FPA, HCO, DJA, RAP, JASN, RL and LFF revised the manuscript. All authors read and approved the manuscript. 
1 Introduction

Crops are attacked by about 67,000 species of organisms, including insects and mites, which are estimated to cause production losses ranging from 10 to $16 \%$ (Ross and Lembi 1985). In order to reduce these losses, new systems have been developed using nanotechnology to protect crops from pests such as weeds, insects, fungi, and mites, as well as to detect and treat plant diseases, deliver fertilizers and other active agents, increase nutrient and water absorption, and allow genetic exploration and transformation (Koul 2019).

Nanobiopesticides are nanomaterials with pesticidal activity or nanostructured carriers loaded with active biological compounds. Such 
(corn earworm) and Tetranychus urticae (Koch) (two-spotted spider mite), together with lower cytotoxicity in 3T3 fibroblasts and V79 lung cells.

Oliveira et al. $(2018 a, 2019)$ used zein to encapsulate combinations of geraniol and R-citronellal, as well as geraniol, eugenol, and cinnamaldehyde. In the first study, encapsulation increased the biological activity of the compounds against $T$. urticae. In the second study, enhanced effects were observed against the same pest and Chrysodeixis includens (Walker). In both cases, there were decreased toxic effects towards nontarget organisms. Kamaraj et al. (2018) demonstrated potential antifeedant activity of neem gum-loaded nanoparticles against $H$. armigera and Spodoptera litura (Fabricius) larvae and pupae, while this nanoformulation did not affect the nontarget organism Eudrilus eugeniae (Kinberg).

Adopting the same approach, Pascoli et al. (2019) prepared neem oilloaded zein nanoparticles with a mean diameter of $278 \pm 6.1 \mathrm{~nm}$, which were stable under the experimental conditions. In vitro ecotoxicological assays showed that the new system decreased or eliminated the toxic effects of the active compound against nontarget organisms such as Allium cepa L. and Caenorhabditis elegans. In addition, the formulation did not affect soil bacteria involved in the nitrogen cycle. However, there have not yet been any tests of the biological activity of this nanoformulation towards target insects, or its potential phytotoxicity under realistic in vivo conditions. 
(Gennadius), and iii) the two-spotted spider mite $T$. urticae as target organisms.

114 The phytotoxic effects of these nanoparticles against Phaseolus vulgaris L. was

also evaluated. The stability of the nanoparticles was investigated during 90 days, using measurements of mean hydrodynamic diameter, polydispersity index, span index, zeta potential, nanoparticle concentration, and encapsulation efficiency. This innovative study opens perspectives for the use of nanobiopesticides based on neem and zein nanoparticles in pest control.

\section{Materials and Methods}

\subsection{Supplies} (catalogue number 9010-66-6) were obtained from Sigma-Aldrich. Neem oil (Azamax) was acquired from UPL Brazil. Absolute Ethanol (code AE07218RA, 99.5\%) was purchased from Labsynth. The 18:1 Liss Rhod PE fluorophore (1,2dipalmitoyl-sn-glycero-3-phosphoethanolamine-N-(lissamine rhodamine B sulfonyl) (ammonium salt)), code 810158, was acquired from Avanti Polar Lipids. Seeds of common bean ( $P$. vulgaris cultivar IPR Curió, Carioca group, register 30616, protection 20130167) were kindly supplied by the Agronomic Institute of Paraná (IAPAR, Londrina, Parana, Brazil). Stored grain beetles (A. obtectus) were obtained from a colony maintained at the Biology Laboratory of São Paulo State University (UNESP, Sorocaba, São Paulo, Brazil). Whitefly (B. tabaci) and two-spotted spider mite ( $T$. urticae) were obtained from colonies maintained at São Paulo State University (UNESP, Jaboticabal, São Paulo, Brazil). Other chemicals, reagents, and solvents used were purchased from local suppliers. 


\subsection{Preparation of neem oil-loaded zein nanoparticles}

Zein nanoparticles were prepared by the anti-solvent precipitation method, described by $\mathrm{Hu}$ and McClements (2014), after treatment of zein as performed by Pascoli et al. (2019). Firstly, zein (2\% w/v) was solubilized in a hydroethanolic solution $(85 \% \mathrm{v} / \mathrm{v})$, under magnetic stirring overnight. The $\mathrm{pH}$ of the zein solution was adjusted to 5.8 , followed by centrifugation for $30 \mathrm{~min}$ at $85750 \mathrm{xg}$, heat treatment at $75{ }^{\circ} \mathrm{C}$ for $15 \mathrm{~min}$, and filtering through a $0.45 \mu \mathrm{m}$ membrane (Millipore). A $100 \mathrm{mg}$ aliquot of neem oil (containing $12 \mathrm{~g} / \mathrm{L}$ of azadirachtin) was added to the zein solution. An aqueous solution of Pluronic F-68 (2\% v/v) was prepared and the $\mathrm{pH}$ was adjusted to 4 . The zein solution containing neem oil was rapidly injected into the Pluronic solution, under magnetic stirring. The colloidal formulation was stirred at room temperature, in order to evaporate the ethanol, and water $(\mathrm{pH} 4.0)$ was added to complete to $20 \mathrm{~mL}$. The final concentration of neem oil in the nanoformulation was $5 \mathrm{mg} / \mathrm{mL}$. In field, neem oil concentration was chosen for the formulation. Control nanoparticles were prepared without neem oil. Labeled nanoparticles, with and without neem oil, were also prepared with addition of rhodamine (18:1 Liss Rhod PE) in the zein solution $(0.05 \% \mathrm{~m} / \mathrm{m}$, relative to the polymer), in order to investigate the interaction between the seed beetles and the formulation (Gott et al. 2014).

\subsection{Physico-chemical stability of the nanoparticles}


159 Determinations of the mean hydrodynamic diameter and the polydispersity index 160 of the nanoparticles were performed by photon correlation spectroscopy, using a 161 ZetaSizer Nano ZS 90 analyzer (Malvern Instruments) at a fixed angle of $90^{\circ}$ and temperature of $25^{\circ} \mathrm{C}$. The same equipment was used to determine the zeta potential, according to the microelectrophoresis method (Grillo et al. 2012). The mean nanoparticle diameter was also determined using NanoSight Nanoparticle LM10 instrument (Malvern Panalytical) and the span index (an indicator of the stability of the formulation, showing the width of the size distribution), was calculated as follows:

$$
\text { Span }=\frac{(D 90-D 10)}{D 50}
$$

where D10, D50, and D90 are the mean diameters corresponding to 10, 50, and $90 \%$ of the particle population, respectively. The particle concentrations in the formulations were also measured using a NanoSight equipped with a $532 \mathrm{~nm}$ laser. The images were collected using a sCMOS camera and were processed using NanoSight v. 2.3 software (Grillo et al. 2014). For these analyses, the samples were diluted 1000 times. The efficiency of encapsulation of the neem oil in the zein nanoparticles was quantified using the ultrafiltration/centrifugation method, with analysis using a UV-Vis spectrophotometer (Cary 50, Varian). The samples were centrifuged using Microcon $10 \mathrm{kDa}$ regenerated cellulose ultrafilters (Millipore), which only allowed passage of the unencapsulated neem. The analytical curve concentration range was from 10 to $200 \mu \mathrm{g} / \mathrm{mL}$ and detection employed a wavelength of $225 \mathrm{~nm}$ (Dubhashi et al. 2013). The encapsulation efficiency was calculated by the difference between the amount of neem initially added and the filtered amount obtained. 


\subsection{Phytotoxicity evaluation using bean plants}

The substrate used for plant growth was clay soil and sand, in a ratio of 1:1 (v:v). The pots and growing trays were kept in the greenhouse of the Center of Biological Sciences of Londrina State University (Londrina, Paraná, Brazil), under natural conditions of air relative humidity and temperature, with $75 \%$ of total environmental photosynthetic photon flux density (PPFD). The soil was enriched with the nutrient solution of Hoagland and Arnon (1950) and was regularly watered. Pre- and post-emergence assays were performed, with the following treatments: water (negative control), zein nanoparticles, neem oil-loaded zein nanoparticles, and neem oil. The concentration adopted in each application of these treatments was the same as that recommended for the commercial product: $5 \mathrm{mg} / \mathrm{mL}$ applied at 100 liters per hectare.

For the post-emergence assay, three applications to the leaves of bean plants were performed, with intervals of 7 days. Each treatment was applied to seven pots, each with three seedlings. At the beginning of the experiment, only the first pair of leaves was fully expanded; hence, all the chlorophyll a fluorescence, gas exchange, and oxidative stress analyses were performed using these leaves. In the pre-emergence assay, the treatments were applied once, directly to the soil of five pots (each with 25 seeds), using amounts equivalent to the three applications of the post-emergence test.

Chlorophyll a fluorescence was measured at the adaxial surfaces of the leaves, using an OS1p fluorometer (Opti-Sciences, Hudson, USA). The maximum quantum yield of photosystem II photochemistry $\left(F_{v} / F_{m}\right)$ was determined as follows: 
208 where $F_{0}$ refers to the minimum, $F_{m}$ to the maximum, and $F_{v}$ to the variable 209 fluorescence of dark-adapted leaves after receiving a saturating pulse of actinic light (Baker, 2008). Gas exchange analyses were performed to determine the light-saturated net photosynthesis $\left(A_{\max }\right)$, using a portable infrared gas analyzer (Model $6400 \mathrm{XT}$, LI-COR Biosciences, Lincoln, USA) connected to a $6 \mathrm{~cm}^{2}$ chamber. The saturating PPFD inside the chamber during the analyses was $1,500 \mu \mathrm{mol} \mathrm{m} \mathrm{m}^{-2} \mathrm{~s}^{-1}$, as determined previously using a light-curve analysis. In the post-emergence assay, the analyses were always carried out two days after application of the treatments to the plants, at the same times (07:30 a.m. for $F_{v} / F_{m}$ and 08:30 a.m. for $\left.A_{\max }\right)$. In the pre-emergence assay, the analyses were performed only at the end of the experiment, at the same time-points described.

Hydrogen peroxide and lipid peroxidation were measured as markers of oxidative stress. For these analyses, $100 \mathrm{mg}$ portions of fresh leaves and roots were ground to a powder in liquid nitrogen, followed by extraction with $1.8 \mathrm{~mL}$ of methanol $+0.2 \%$ trichloroacetic acid (TCA). After centrifugation (13700 xg for 5 $\min$ at $4^{\circ} \mathrm{C}$ ), the supernatant was used for measurement of the hydrogen peroxide content by reaction with potassium iodide, in phosphate buffer (Alexieva et al. 2001), and for the determination of thiobarbituric acid reactive substances (TBARS) (Camejo et al. 1998). For determination of hydrogen peroxide, the supernatant was subjected to reaction for one hour with $1 \mathrm{M}$ potassium iodide (KI), in $\mathrm{pH} 7.5$ phosphate buffer (PBS), keeping the mixture on ice and in the dark. at $390 \mathrm{~nm}$, using a 96-well plate and a microplate reader (Model Victor TM 3, 
231 PerkinElmer, Turku, Finland). For determination of TBARS, the supernatant was

232 subjected to reaction with $0.02 \%$ butylated hydroxytoluene $(\mathrm{BHT})$ in $\mathrm{pH} 7.4 \mathrm{PBS}$

233 buffer, together with $1.3 \%$ thiobarbituric acid (TBA) and $0.3 \%$ sodium hydroxide

$234(\mathrm{NaOH})$, in the presence of $50 \% \mathrm{TCA}$, at $60{ }^{\circ} \mathrm{C}$ for $60 \mathrm{~min}$. Lipid peroxidation

235 concentration was determined using a malondialdehyde (MDA) standard curve

236 constructed from fluorescence readings obtained at excitation and emission

237 wavelengths of 535 and $590 \mathrm{~nm}$, respectively, employing the Victor TM 3 reader

238 (Camejo et al. 1998).

239 Finally, for plant dry mass determination, the plants were harvested (after

24013 days in the pre-emergence assay and after 24 days in the post-emergence 241 assay), individually packed in paper bags, and dried in an oven at $60{ }^{\circ} \mathrm{C}$ until 242 reaching constant mass.

\section{$243 \quad 2.5$ Biological activity assays}

\subsubsection{Evaluation of mortality of Acanthoscelides obtectus and its} interaction with the nanobiopesticide

The bioassays using $A$. obtectus were conducted in the Biology Laboratory of UNESP/ICTS, in controlled climate chambers with constant aeration, absence of light, temperature of $27 \pm 2{ }^{\circ} \mathrm{C}$, and maximum and minimum humidity of 73 and $52 \%$, respectively, based on the studies of Jumbo et al. (2014), Soares et al. (2014), and Janković-Tomanić et al. (2015). The colony was maintained under the same conditions. The Phaseolus vulgaris (Qualitá ${ }^{\circledR}$ ) used to maintain the culture and to carry out the experiments was previously kept in a freezer for 14 days and dried, in order to prevent possible infestation from the field and to 
254 reduce any potential effect of insecticide residue, as proposed by Jumbo et al. 255 (2014).

The biocidal activity assays were carried out according to the method described by Jumbo et al. (2014), using an acute mortality assay (96 h) to estimate the mean lethal concentration $\left(\mathrm{LC}_{50}\right)$. Masses of $25 \mathrm{~g}$ of beans were placed in $145 \mathrm{~mL}$ plastic bottles with small holes in the cap for aeration, followed by application of the treatments (zein nanoparticles, neem oil-loaded zein nanoparticles and neem oil) and shaking the vials manually for 60 seconds to ensure complete distribution of the material in the beans. Ten unsexed adults (1 to 5 day old) of $A$. obtectus were placed in each vial. The experiment was carried out with concentrations equivalent to $1.35,2.7,5.4,10.8$, and $21.6 \mathrm{mg}$ of azadirachtin per $\mathrm{kg}$ of beans (Tofel et al. 2017), using 0.5, 1.12, 2.25, 4.5 and 9 $\mathrm{mL}$ of formulation, respectively. After the exposure period, mortality was evaluated using a stereomicroscope (Model XTB-2B, Coleman), with the beetles being considered dead when they did not show movement, even when stimulated by touching with a fine-bristle brush for $4 \mathrm{~min}$. Two replicates were performed for each dose and for the control treatment, and the experiment was repeated three times. The LC50 values were estimated as proposed by Hamilton et al. (1977), using the Trimmed Spearman-Karber method.

The treatments with the rhodamine-labeled nanoparticles were performed in the same way, under the same experimental conditions as described for the $A$. obtectus biological activity assay, using the $\mathrm{LC}_{50}$ concentration for the neem oilloaded nanoparticles and the same volume for the zein nanoparticles without the active agent. The insetcs were analyzed at the Central Multiusers Laboratory of the School of Agricultural Sciences (UNESP) after $96 \mathrm{~h}$ of exposure, using a Carl 
279 Zeiss SteREO Discovery v. 12 microscope fitted with a red filter for fluorescence,

280 in order to identify the presence of the nanoformulation in the bodies of the 281 insects. The images were acquired with an Axiocam 2.0 Zen Blue camera and 282 were treated using the equipment software. The images of the bodies of $A$. obtectus were merged with the fluorescence evaluation images, enabling 284 visualization of the interactions between the beetles and the treatments. A total of 10 specimens were analyzed for each treatment. Untreated control specimens were used to evaluate any possible natural fluorescence emitted by the body of the insect.

\subsubsection{Bemisia tabaci mortality assay}

The whitefly (B. tabaci) mortality experiments were conducted in the Microbial Control of Pest Arthropods Laboratory (UNESP/FCAV). The whiteflies used in this assay were reared on bean plants in a greenhouse and were collected in flat bottom glass tubes, using manual suction. A total of 480 insects were collected in 48 tubes (10 insects per tube). These tubes were transferred to the previously treated bean plants in pots (24 pots, each with 2 plants) and were left open until the flies had emerged from the tubes. Prior to the transfer of the whiteflies, the treatments were applied to the bean plants by manual spraying, as recommended by the manufacturer of the commercial neem oil (3 applications, spaced at intervals of 7 days). Three scenarios with different concentrations were simulated: concentration of $5 \mathrm{mg} / \mathrm{mL}, 100 \mathrm{~L} /$ hectare (also as recommended by the manufacturer), concentration estimating overdosage (15 mg/mL, 100 
$\mathrm{mg} / \mathrm{mL}, 100 \mathrm{~L} /$ hectare). Six replicates were performed for each treatment and the dead insects found on the floors of the cages were counted daily.

\subsubsection{Biological effects on Tetranychus urticae}

The assays of biological effects against the $T$. urticae mite were conducted in the Acarology Laboratory (UNESP/FCAV), using mites obtained from jack bean plants (Canavalia ensiformes L.). The plants were cultivated in $2 \mathrm{~L}$ pots containing soil, sand, and bovine manure $(1: 1: 1, \mathrm{v}: \mathrm{v}: \mathrm{v})$ as the substrate. The mites were kept in a temperature-controlled climate chamber at $25 \pm 1 \stackrel{\circ}{ } \mathrm{C}$, relative humidity (RH) of $60 \pm 10 \%$, and $12 \mathrm{~h} / 12 \mathrm{~h}$ light/dark photoperiod. The experiments were performed using arenas ( $2.5 \mathrm{~cm}$ diameter) of $C$. ensiformes leaves obtained using a circular metal cutter. The arenas were placed in Petri dishes $(9 \times 2 \mathrm{~cm})$ containing a moistened foam and a hydrophilic cotton layer $(1.0 \mathrm{~cm})$, in order to maintain the turgidity of the arenas, and were surrounded with hydrophilic cotton to avoid escape of the mites.

Evaluations of biological activity were performed using the larvae, nymphs, and adults of $T$. urticae. The treatments (water as the negative control, zein nanoparticles, zein nanoparticles with neem oil at $5 \mathrm{mg} / \mathrm{mL}$, neem oil at $5 \mathrm{mg} / \mathrm{mL}$, and the commercial synthetic acaricide Oberon $^{\circledR}$ as a positive control) were evaluated for direct and residual action. For evaluation of the direct action, the mites in the different stages of development (larvae, nymphs, or adult females) were transferred to the arenas (10 mites per arena). The treatments were then

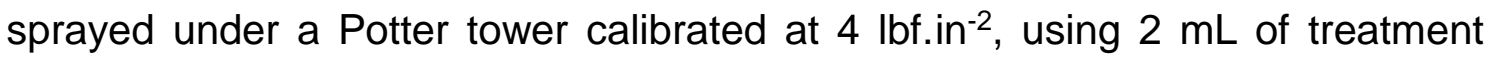
solution per arena, corresponding to $1.56 \mathrm{mg} . \mathrm{cm}^{-2}$ of dry residue. Each treatment 
was repeated 8 times. After the applications, the arenas were transferred to a climate-controlled chamber, as described above. For the residual evaluation of the formulations, jack bean (C. ensiformes) seeds were planted in $5 \mathrm{~L}$ pots containing soil, sand, and bovine manure (1:1:1, v:v:v) as substrate. Approximately 30 days after germination, the plants were separated into 5 groups of three plants to receive the applications of the different treatments. The products were applied with a $500 \mathrm{~mL}$ capacity manual sprayer, until complete coverage of the plants. An average of $15 \mathrm{~mL}$ of treatment solution was required per plant. After 1,6 , and 12 days following the applications, leaves of the bean plants were collected and arenas were prepared in Petri dishes, as described above, followed by the transfer of 10 larvae, nymphs, or adults to each arena. Each assay employed 8 replicates. The numbers of mites that were alive, dead, or trapped in the cotton barrier were counted daily during 5 days, using a stereomicroscope (40x magnification). Mites that did not react to the touch of a fine brush were considered dead.

\subsection{Statistical analysis}

The results of the biological activity assays were treated as proposed by Abbott (1925) for corrected mortality. The statistical analyses were performed with GraphPad Prism v. 6 software, using one-way ANOVA for stability, two-way ANOVA for phytotoxicity and biological activity assays against Acanthoscelides obtectus and Tetranychus urticae, and repeated measures ANOVA for Bemisia tabaci mortality followed by the Tukey post-hoc test, at a significance level of $p<0.05$. 


\section{Results}

\subsection{Physico-chemical stability of the nanoparticles}

In this study, the physico-chemical stability of the neem oil-loaded zein nanoparticles was evaluated by determination of several parameters during storage of the formulations for 90 days. Initially, mean diameter (Figure 1A) was obtained by DLS $(288 \pm 6 \mathrm{~nm})$ and it showed a significant increase on day 60 reaching an average diameter of $313 \pm 8.1 \mathrm{~nm}(F=15.54, D F=5, P<0.0001)$.

Using the same technique, the polydispersity index (Figure 1A) was found to remain at around 0.2 , with a decrease on day $10(F=7.387, D F=5, P=0.0022)$. and no other significant differences between day 10 and 90, indicating good physicochemical stability of the polymer system. Use of the NTA technique, which enables determination of the hydrodynamic diameter of the particles by directly measuring their diffusion coefficients when they are in Brownian motion, resulted in nanobiopesticide particle sizes that were smaller than obtained by DLS, with $198 \pm 16 \mathrm{~nm}$ (Figure 1B). Using this technique, the mean diameters oscillated significantly, increasing on day 20 and 60 and decreasing on day 10 and $90(F=$ 59.17, $D F=5, P<0.0001)$ throughout the storage time, which could have been because the technique is more sensitive and analyzes each particle individually.

The span index values (Figure 1B) were less than 1 and showed significant decrease only on day $10(F=7.387, D F=5, P=0.0022)$. No other significant differences during the 90 days of storage were observed, which is also a characteristic of stable formulations. 
significant fluctuations during the 90 days of storage $(F=172.5, D F=5, P<$

a)

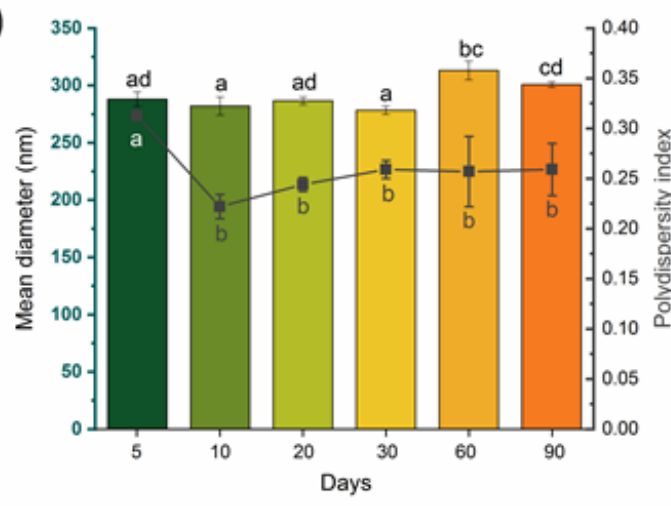

c)

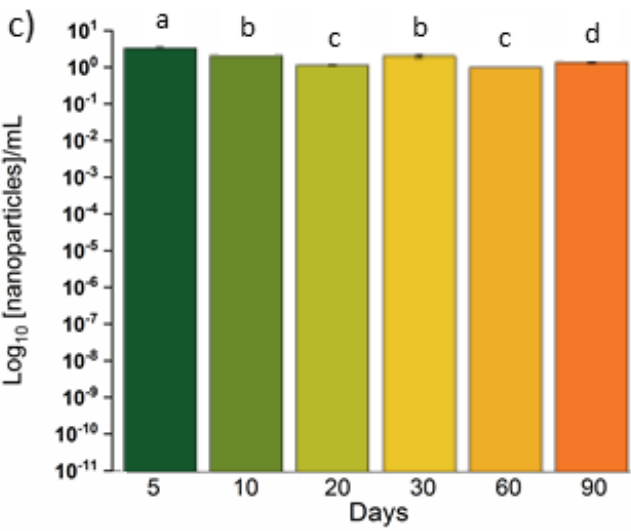

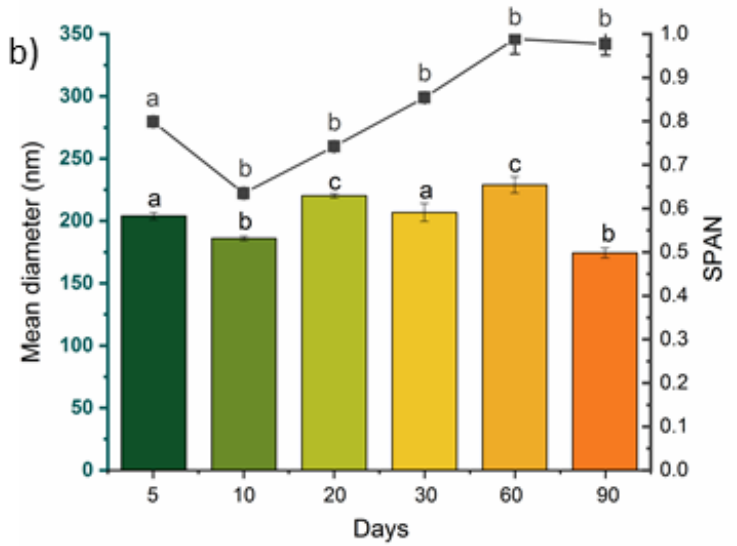

d)

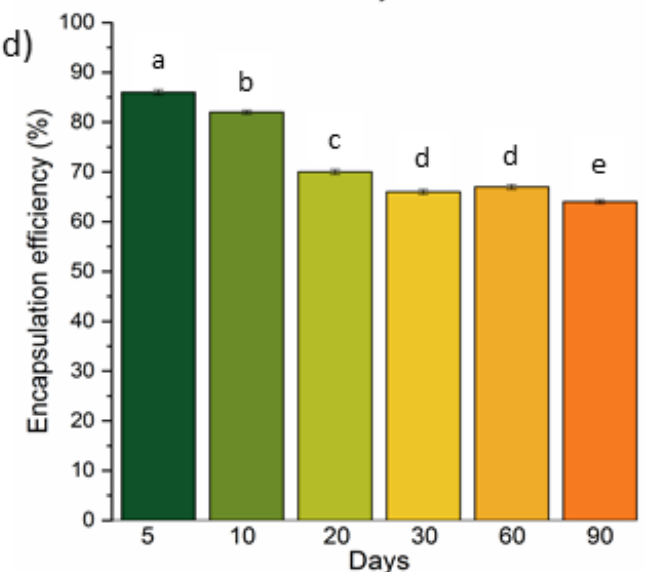

Fig. 1 Stability of the neem oil-loaded zein nanoparticles during 90 days: A) Mean hydrodynamic size (bars) and polydispersity index (line), obtained using DLS. B) Mean hydrodynamic size (bars) and span index (line), obtained using NTA. C) 
383 Concentration of nanoparticles in the formulation, obtained by NTA. D)

384 Encapsulation efficiency of neem oil in the zein nanoparticles, obtained by UV-

385 Vis spectroscopy. The data are expressed as the average of three independent experiments $(n=3)$ and the error bars represent the standard deviations. Equal letters indicate values that do not differ significantly according to one-way ANOVA followed by the Tukey post-hoc test $(p<0.05)$.

The zeta potential values (data not shown) oscillated significantly during the 90 days of storage $-36 \pm 1 \mathrm{mV}$ on day $1,-41 \pm 2.9 \mathrm{mV}$ on day $10,-24.6 \pm 0.8$ $\mathrm{mV}$ on day 20 and $-15.5 \pm 2.5 \mathrm{mV}$ on day $60(F=86.41, D F=5, P<0.0001)$ indicating a lack of stability. However, Pluronic F-68 was used during the nanoparticles preparation process, which provided steric hindrance and was responsible for the stability of the system.

\subsection{Phytotoxicity evaluation using bean plants}

The $F_{v} / F_{m}$ ratio, which indicates the maximum quantum efficiency of electron transport in photosystem II, was not affected by any of the formulations tested, regardless of the type of treatment (Table 1). All the leaves presented $F_{v} / F_{m}$ values near 0.8 . The $A_{\max }$ values for the treated plants showed no significant differences, compared to the corresponding controls, evidencing that 402 the formulations did not affect photosynthetic activity in the leaves. In the third evaluation of the plants in the post-emergence test, there was a significant decrease of $A_{\max }$, relative to the first and second evaluation of the same plants ( $F$ 
407 for the analyses.

Similar to the photosynthetic parameters, lipid peroxidation and hydrogen peroxide levels in the roots and leaves showed no significant differences between

410 the control and the treatments (Table 1), demonstrating that the formulations did not induce oxidative stress in common bean plants.

413 Table 1 Maximum quantum yield of photosystem II photochemistry $\left(F_{v} / F_{m}\right)$, lightsaturated net photosynthesis $\left(A_{\max }\right)$, and oxidative stress parameters of the bean plants. $1^{\text {st }}, 2^{\text {nd }}$, and $3^{\text {rd }}$ represent the analyses after the first, second, and third treatment applications, respectively. The data are expressed as average \pm 417 standard deviation for three $(n=3)$ analyses using ten (10) and fourteen (14) plants for the pre- and post-emergence assays, respectively. The symbols $\dagger$ and $\phi$ indicate significant difference relative to the $1^{\text {st }}$ and $2^{\text {nd }}$ analyses, respectively, according to two-way ANOVA followed by the Tukey post-hoc test $(p<0.05)$.

\begin{tabular}{|c|c|c|c|c|c|c|}
\hline \multirow[b]{2}{*}{ Treatments } & \multirow[t]{2}{*}{$F_{v} / F_{m}$} & \multirow[t]{2}{*}{$\begin{array}{c}\mathrm{A}_{\max }\left(\mu \mathrm{mol} \mathrm{CO}_{2}\right. \\
\left.\mathrm{m}^{-2} \mathrm{~s}^{-1}\right)\end{array}$} & \multicolumn{2}{|c|}{ 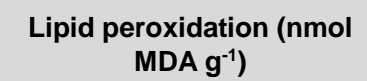 } & \multicolumn{2}{|c|}{$\mathrm{H}_{2} \mathrm{O}_{2}\left(\mu \mathrm{mol} \mathrm{g} \mathrm{g}^{-1}\right)$} \\
\hline & & & root & leaf & root & leaf \\
\hline \multicolumn{7}{|c|}{ Pre-emergence assay } \\
\hline Control & $0.774 \pm 0.011$ & $15.8 \pm 3.3$ & $12.7 \pm 2.8$ & $29.2 \pm 5.8$ & $31.0 \pm 2.3$ & $332.2 \pm 12.3$ \\
\hline Zein NP & $0.760 \pm 0.021$ & $16.5 \pm 2.0$ & $9.6 \pm 3.2$ & $36.6 \pm 3.0$ & $35.3 \pm 3.4$ & $356.1 \pm 19.5$ \\
\hline Neem NP & $0.753 \pm 0.015$ & $17.5 \pm 2.3$ & $7.2 \pm 4.4$ & $32.8 \pm 9.1$ & $25.3 \pm 4.8$ & $334.9 \pm 40.4$ \\
\hline Neem & $0.767 \pm 0.019$ & $16.2 \pm 2.0$ & $12.6 \pm 8.8$ & $33.8 \pm 5.9$ & $27.9 \pm 4.2$ & $356.7 \pm 33.0$ \\
\hline \multicolumn{7}{|c|}{ Post-emergence assay } \\
\hline $1^{\text {st }}$ Control & $0.826 \pm 0.008$ & $25.9 \pm 3.1$ & - & - & - & - \\
\hline $1^{\text {st }}$ Zein NP & $0.827 \pm 0.007$ & $25.6 \pm 3.0$ & - & - & - & - \\
\hline $1^{\text {st }}$ Neem NP & $0.829 \pm 0.006$ & $23.5 \pm 1.6$ & - & - & - & - \\
\hline
\end{tabular}




\begin{tabular}{l|l|l|c|c|c|c}
$\mathbf{1}^{\text {st }}$ Neem & $0.830 \pm 0.005$ & $26.5 \pm 2.5$ & - & - & - & - \\
$\mathbf{2}^{\text {nd }}$ Control & $0.794 \pm 0.015$ & $16.7 \pm 2.4$ & - & - & - & - \\
$\mathbf{2}^{\text {nd }}$ Zein NP & $0.792 \pm 0.019$ & $17.3 \pm 1.1$ & - & - & - & - \\
$\mathbf{2}^{\text {nd }}$ Neem NP & $0.788 \pm 0.008$ & $17.1 \pm 2.2$ & - & - & - & - \\
$\mathbf{2}^{\text {nd }}$ Neem & $0.791 \pm 0.020$ & $16.75 \pm 1.7$ & - & - & - & $362.7 \pm 39.8$ \\
$\mathbf{3}^{\text {rd }}$ Control & $0.790 \pm 0.017$ & $4.1 \pm 2.1 \dagger^{\phi}$ & $12.7 \pm 4.6$ & $47.5 \pm 5.8$ & $28.2 \pm 16.2$ & \\
$\mathbf{3}^{\text {rd }}$ Zein NP & $0.785 \pm 0.019$ & $5.2 \pm 2.8 \dagger^{\phi}$ & $12.3 \pm 5.5$ & $48.3 \pm 4.8$ & $18.3 \pm 12.5$ & $373.9 .1 \pm 40.2$ \\
$\mathbf{3}^{\text {rd }}$ Neem NP & $0.808 \pm 0.005$ & $6.6 \pm 3.1 \dagger^{\phi}$ & $14.2 \pm 4.3$ & $50.1 \pm 3.0$ & $25.60 \pm 20.5$ & $450.9 \pm 48.9$ \\
$\mathbf{3}^{\text {rd }}$ Neem & $0.797 \pm 0.014$ & $5.8 \pm 2.8 \dagger^{\phi}$ & $7.8 \pm 3.4$ & $51.0 \pm 5.5$ & $12.3 \pm 10.3$ & $422.8 \pm 44.4$
\end{tabular}

423 analyses, the dry mass of the bean plants did not show any significant difference 424 among the control and the treatments in the pre- and post-emergence 425 experiments. This demonstrates that the biopesticide and the neem oil did not 426 affect the growth of the plants under the experimental conditions adopted (Figure 427 2). 


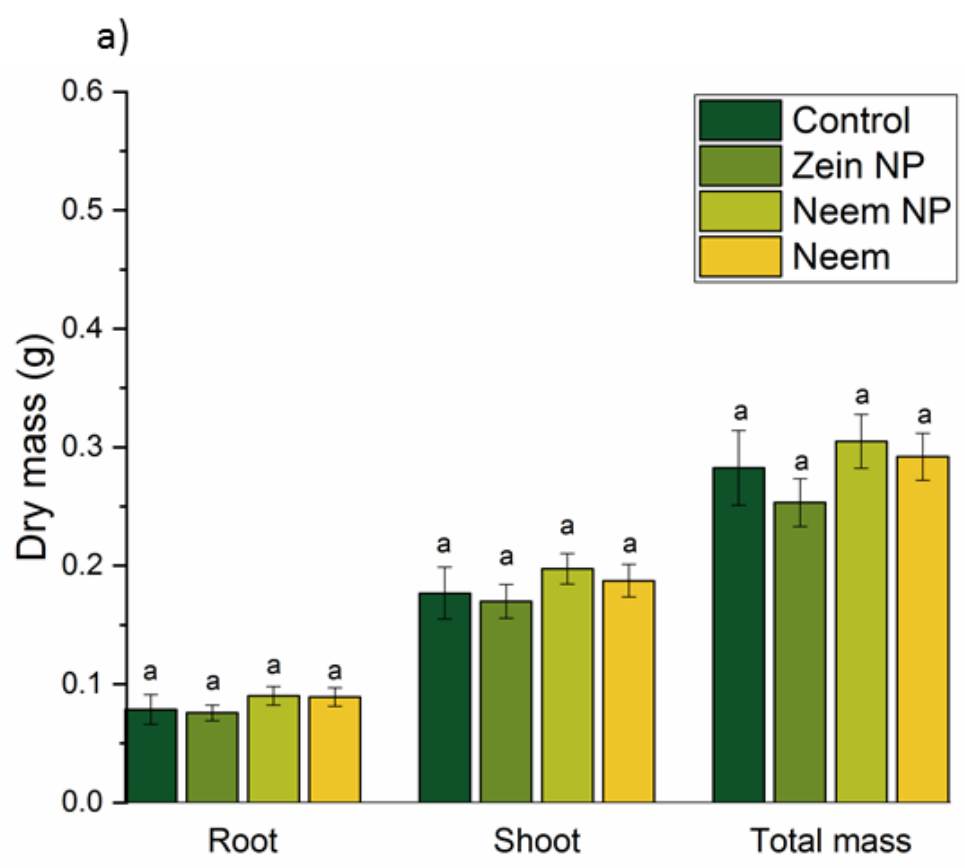

b)

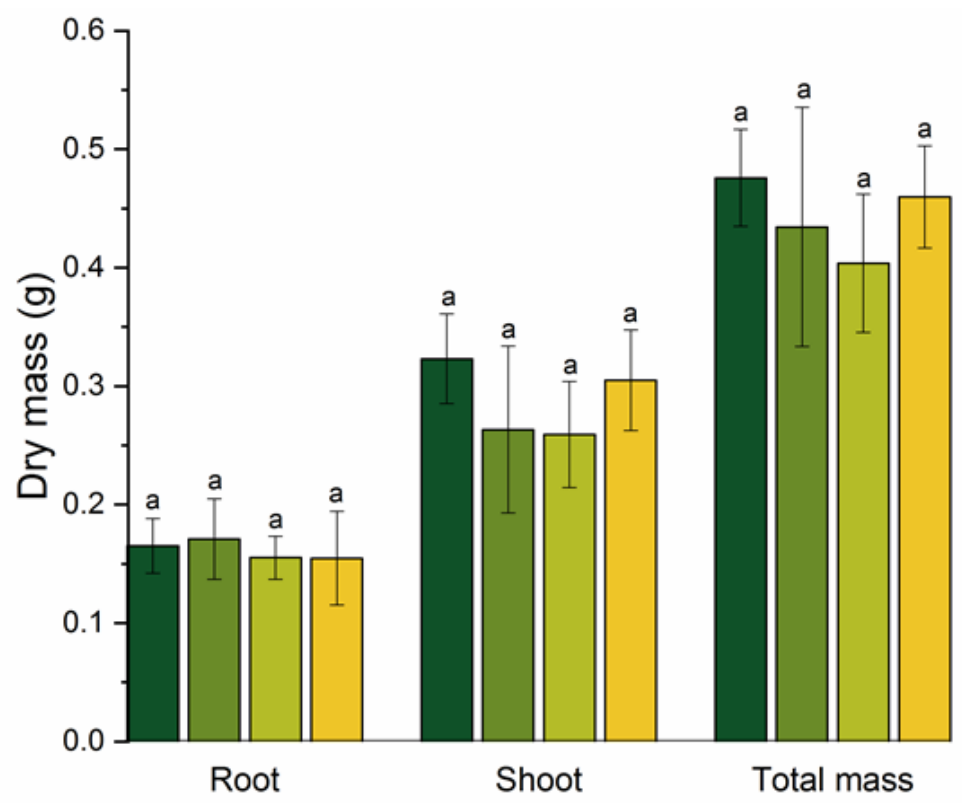

Fig. 2 Results of phytotoxicity assays using common bean plants: Dry masses of 431 plants treated with water (control), zein nanoparticles (Zein NP), neem oil-loaded 432 zein nanoparticles (Neem NP), and neem oil (Neem). A) Pre-emergence assay; 433 B) post-emergence assay. The data are expressed as averages of ten $(n=10)$ 434 and fourteen $(n=14)$ plants for the pre- and post-emergence assays, 435 respectively. The error bars represent the standard deviations. Equal letters 
437 followed by the Tukey post-hoc test $(\mathrm{p}<0.05)$.

\subsection{Biological activity}

\subsubsection{Biological activity against Acanthoscelides obtectus}

441

In the $A$. obtectus acute assays, the LC 50 was estimated by the Trimmed

442

Spearman-Karber method, according to the confidence interval of the results.

443

The LC50 values were $6.65 \mathrm{mg}$ of azadirachtin per $\mathrm{kg}$ of beans for the neem oil-

444

loaded zein nanoparticles and $11.22 \mathrm{mg}$ of azadirachtin per $\mathrm{kg}$ of beans for the neem oil, indicating that the new system provided greater efficiency against this

The results (Figure $3 \mathrm{~A}$ ) showed that the neem oil nanobiopesticide caused significant mortality of the pest from the second lowest concentration tested, while the neem oil only caused significant mortality at the highest concentration evaluated. It was also observed that the zein nanoparticles without the active compound only had an effect at the highest concentration employed $(F=24.00$, $D F=3, P<0.0001)$

In order to evaluate the contact between the nanobiopesticide and the 454 insects, the nanobiopesticide was labeled with the 18:1 Liss Rhod PE 455 fluorophore. The resulting material had the same physical chemical characteristics as the unlabeled nanobiopesticide (data not shown). 
458 exposure of the $A$. obtectus individuals to the nanoformulations was mainly via 459 the integument (Figure 3B), with the greatest exposure occurring in the ventral 460 region, especially the legs and mouthparts. Nanoparticles could also be seen on 461 the antennae and the abdomen. These results suggested that the increased 462 mortality of $A$. obtectus (Figure 3A) was probably due to direct contact and 463 interaction with the nanobiopesticide, with better adhesion facilitating absorption 464 of the nanostructures by the insect. 


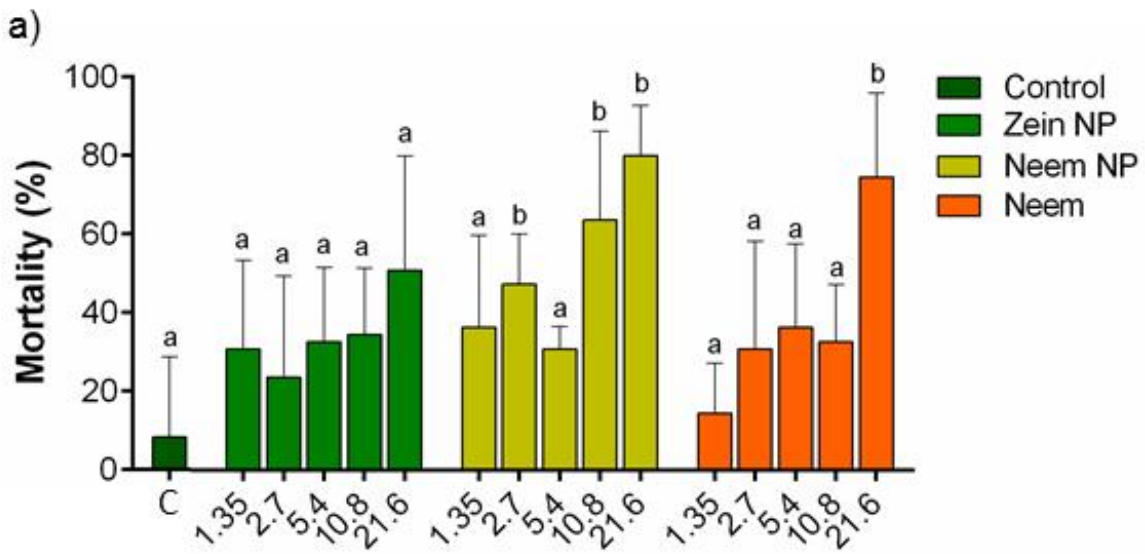

b)

Concentration ( $\mathrm{mg}$ azadirachtin/ $\mathrm{kg}$ bean)
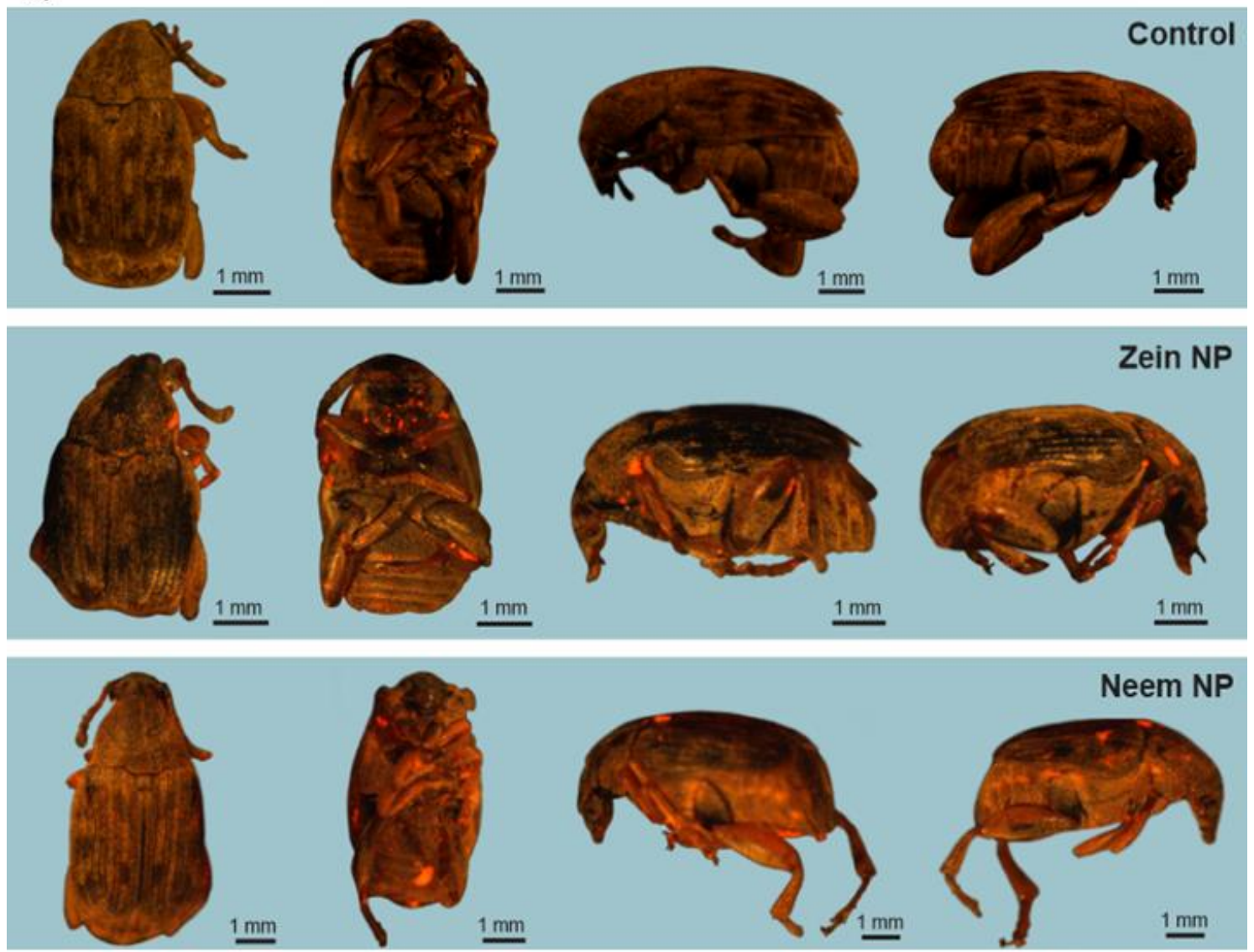

467 Fig. 3 Results of assays using Acanthoscelides obtectus: A) Mortality of $A$. 468 obtectus following acute exposure $(96 \mathrm{~h})$ to beans treated with the zein 469 nanoparticles (Zein NP), the neem oil-loaded zein nanoparticles (Neem NP), and 470 the neem oil (Neem), at concentrations of $1.35,2.7,5.4,10.8$, and $21.6 \mathrm{mg}$ of 
471 azadirachtin per $\mathrm{kg}$ of beans. The zein nanoparticle treatment was used as a

472 control, at the same volume as the treatments containing the active agent. B)

473 Images of $A$. obtectus exposed for $96 \mathrm{~h}$ to beans treated with neem oil-loaded

474 zein nanoparticles labeled with rhodamine (Neem NP), at a concentration of 6.64

$475 \mathrm{mg}$ of azadirachtin per $\mathrm{kg}$ of beans. Labeled zein nanoparticles and untreated

476 bruchines were used as a control. The data are expressed as the average of

477 three independent experiments $(n=3)$, normalized to \%. The error bars represent

478 the standard deviation. Equal letters indicate values that do not differ significantly

479 according to two-way ANOVA followed by the Tukey post-hoc test $(p<0.05)$.

480

481

\subsubsection{Biological effect on Bemisia tabaci}

Figure 4 shows the results of the mortality assays using the nanoformulations and neem oil against $B$. tabaci. The treatments were performed 484 at concentrations of $5 \mathrm{mg} / \mathrm{mL}$, as recommended by the manufacturer of commercial neem oil, $15 \mathrm{mg} / \mathrm{mL}$, representing overdosage, and $1 \mathrm{mg} / \mathrm{mL}$, representing less use of the bioinsecticide.

In the assay performed under the use conditions recommended by the manufacturer (Figure 4A), the mortality of the pest presented significant increases, compared to the control, starting on the $3^{\text {rd }}$ day for the neem oil, and on the $5^{\text {th }}$ day for the zein nanoparticles with neem oil. In this case, the commercial neem oil showed no higher efficiency than the neem oil-loaded zein nanoparticles $(F=7.22, D F=18, P<0.0001)$. 

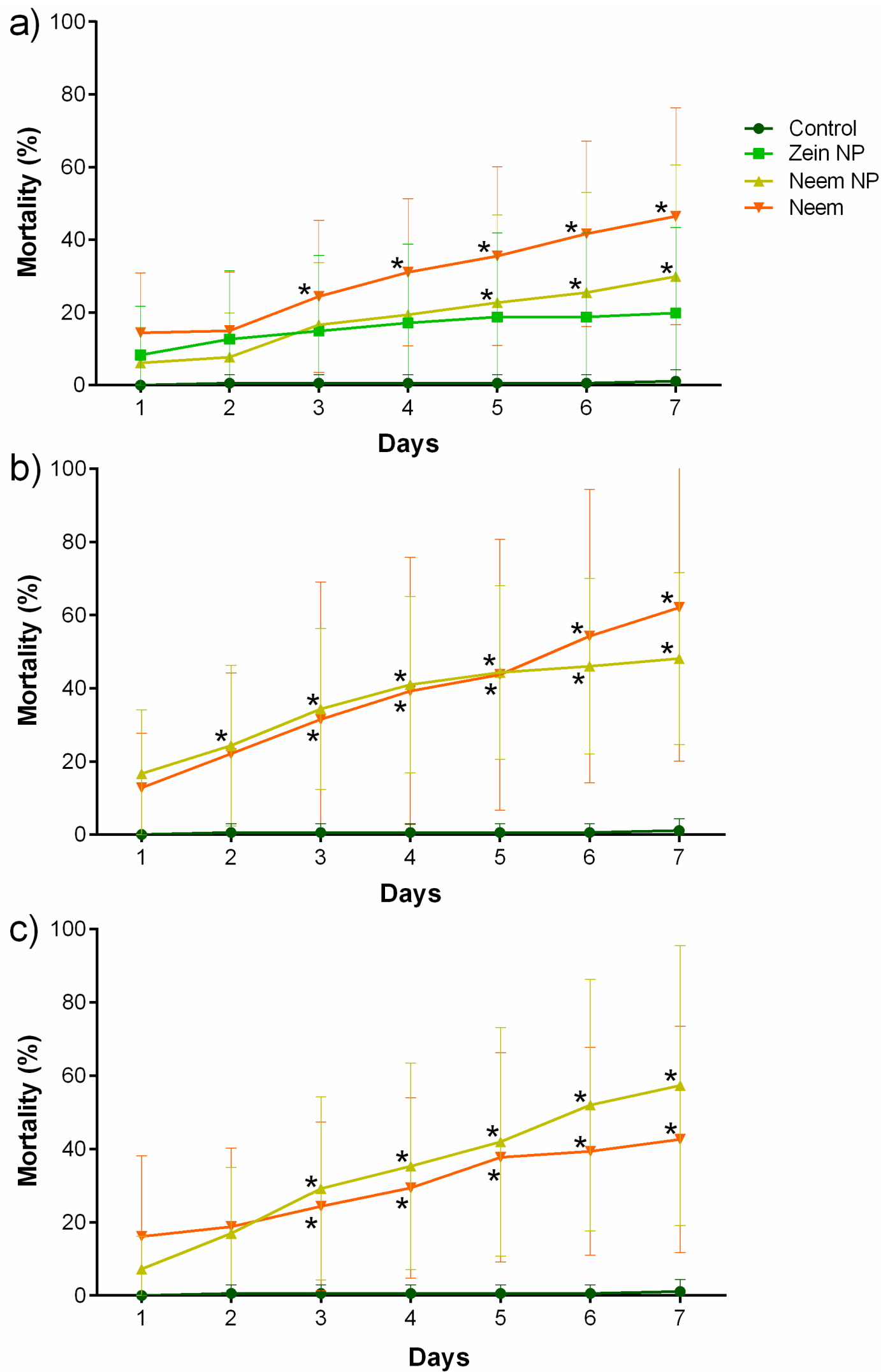

494

Fig. 4 Mortality of whiteflies treated with zein nanoparticles (Zein NP), neem oil496 loaded zein nanoparticles (Neem NP), and neem oil (Neem), at A) the 
497 recommended concentration (5 mg/mL), B) overdosage concentration (15 $498 \mathrm{mg} / \mathrm{mL})$, and C) lower dosage $(1 \mathrm{mg} / \mathrm{mL})$. The data are expressed as averages 499 of three independent experiments $(n=3)$, normalized to \%. The error bars 500 represent the standard deviation. The symbol * indicate significant difference 501 relative to control. A significance level of $P<0.05$ was adopted using repeated 502 measures ANOVA followed by the Tukey post-hoc test.

503

In the overdosage scenario (Figure 4B), the treatments presented significantly higher mortality compared to the control from day 2 to day $7(F=$ 10.46, $D F=12, P<0.0001)$, with no significant difference between the treatments. Considering the capacity of $B$. tabaci to develop resistance to pesticides, the increase in mortality could be attributed to the increase of the concentration of the applied active compound.

In the assay using lower concentrations of the bioinsecticide (Figure 4C),

511 the mortality results were again similar for the neem oil and the neem oil-loaded nanoparticles, and significantly higher compared to the control from day $3(F=$ 16.65, $D F=12, P<0.0001)$. However, calculation of the areas under the curves

514 (Table 2) revealed that in the experiment carried out using the neem oil at a 515 concentration of $1 \mathrm{mg} / \mathrm{mL}$, the nanobiocide and the neem oil showed the same result with areas of 207.7 and 179, respectively, showing the potential for using

517 a lower concentration of the pesticide to control whitefly.

519 Table 2 Area under the curve values for the biological activity assays using the 520 control and the nanobiopesticide at concentrations of 5,15 , and $1 \mathrm{mg} / \mathrm{mL}$ : water 
521 (Control), zein nanoparticles (Zein NP), neem-loaded zein nanoparticles (Neem

$522 \mathrm{NP}$ ), and neem oil (Neem). The data are expressed as the average \pm standard 523 deviation of three independent experiments $(n=3)$. Different letters denote 524 significant differences. A significance level of $P<0.05$ was adopted using two525 way ANOVA followed by the Tukey post-hoc test.

\begin{tabular}{|c|c|}
\hline Treatments & $\begin{array}{c}\text { Area under the curve } \\
\text { (mortality } x \text { days) }\end{array}$ \\
\hline \multicolumn{2}{|c|}{$5 \mathrm{mg} / \mathrm{mL}$ assay } \\
\hline Control & $3.33 \pm 4.06^{\mathrm{a}}$ \\
\hline Zein NP & $96.37 \pm 36.72^{b}$ \\
\hline Neem NP & $110.00 \pm 36.61^{b}$ \\
\hline Neem & $178.10 \pm 38.39 b$ \\
\hline \multicolumn{2}{|c|}{$15 \mathrm{mg} / \mathrm{mL}$ assay } \\
\hline Neem NP & $222.40 \pm 39.45^{b}$ \\
\hline Neem & $228.10 \pm 60.02^{b}$ \\
\hline \multicolumn{2}{|c|}{$1 \mathrm{mg} / \mathrm{mL}$ assay } \\
\hline Neem NP & $207.70 \pm 48.28^{b}$ \\
\hline Neem & $179.00 \pm 44.36^{b}$ \\
\hline
\end{tabular}

526

\subsubsection{Tetranychus urticae mortality}

Figure 5 shows the mortality rates following direct application of the 529 treatments (at a neem oil concentration of $5 \mathrm{mg} / \mathrm{mL}$ ) to the larvae (Figure $5 \mathrm{~A}$ ), nymphs (Figure 5B), and adults (Figure 5C). For the larvae and nymphs, use of

531 the neem oil-loaded nanoparticles led to a slightly higher mortality rate, compared 532 to use of the neem oil, although the differences were not significant. However, 533 both neem oil and the neem oil-loaded zein nanoparticles showed acaricide potential against $T$. urticae, exceeding $50 \%$ mortality, with a similar result for the positive control $(F=1.09, D F=3, P=0.3684$ and $F=3.08, D F=3, P=0.0436$, 
536 respectively). It was interesting to note that the zein nanoparticles caused mortality of the mites, especially when applied to the larvae, where the mortality rates were similar to those observed for the insecticide.

The residual treatments resulted in similar response profiles for the larvae 540 (Figure 5D), nymphs (Figure 5E), and adults (Figure 5F), with the mortality rates generally decreasing over time $(F=23.06, D F=11, P<0.0001, F=66.34, D F=$ 11, $P<0.0001$ and $F=38.41, D F=11, P<0.0001$, respectively). The most efficient results were observed on the first day after application $(F=31.33, D F=$ 3, $P<0.0001, F=19.67, D F=3, P<0.0001$ and $F=11.68, D F=3, P<0.0001$, respectively), which were comparable to the results obtained in the direct treatment (Figures $5 \mathrm{~A}, 5 \mathrm{~B}$, and $5 \mathrm{C}$ ). A possible explanation for this was that in the case of the residual treatment (Figures $5 \mathrm{D}, 5 \mathrm{E}$, and $5 \mathrm{~F}$ ), the leaves were attached to the plants at the time of application, so the active metabolism could have led to the treatments reaching the leaves, resulting in the mites ingesting more of the active ingredient. However, over time, the compounds were degraded and their efficiencies decreased.

An exception to the reduction in mortality over time in the residual effect assays was observed for the effect of the neem nanoparticles on the larvae (Figure 5D), where larval mortality increased on the $12^{\text {th }}$ day. This could be attributed to the ability of the nanoparticles to protect the active agent, hence prolonging its effectiveness, under the experimental conditions employed. 

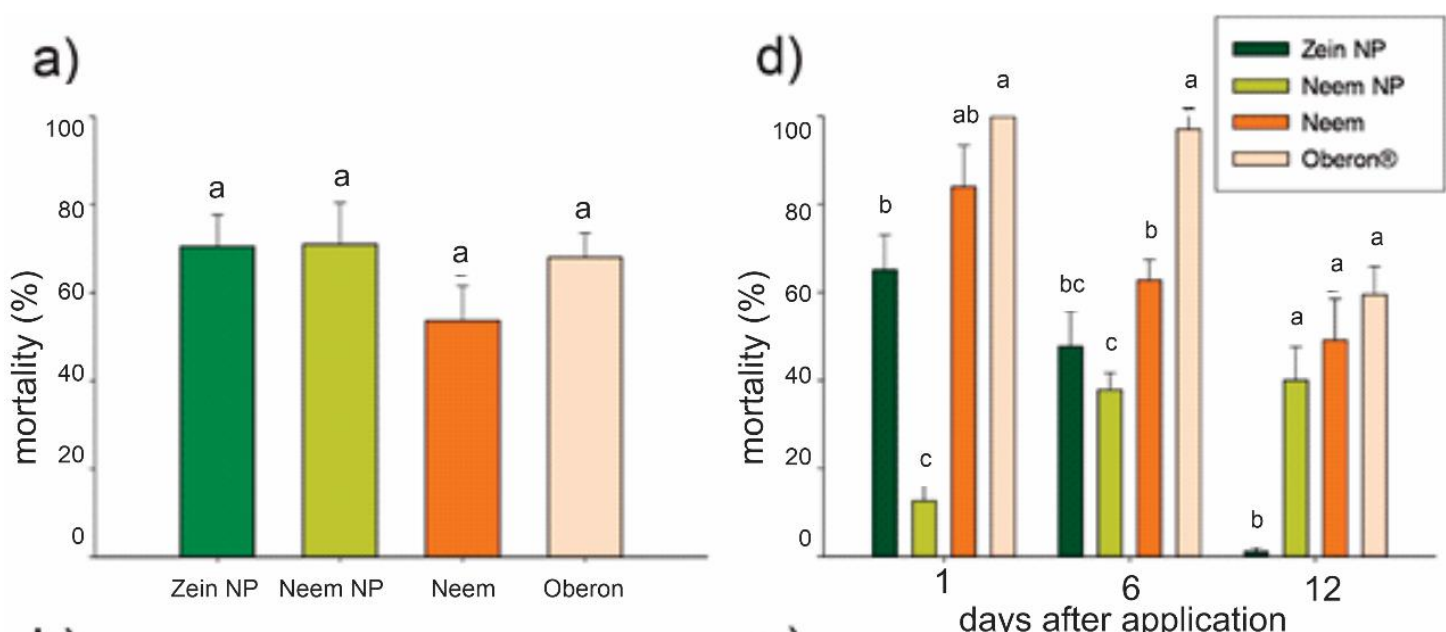

b)
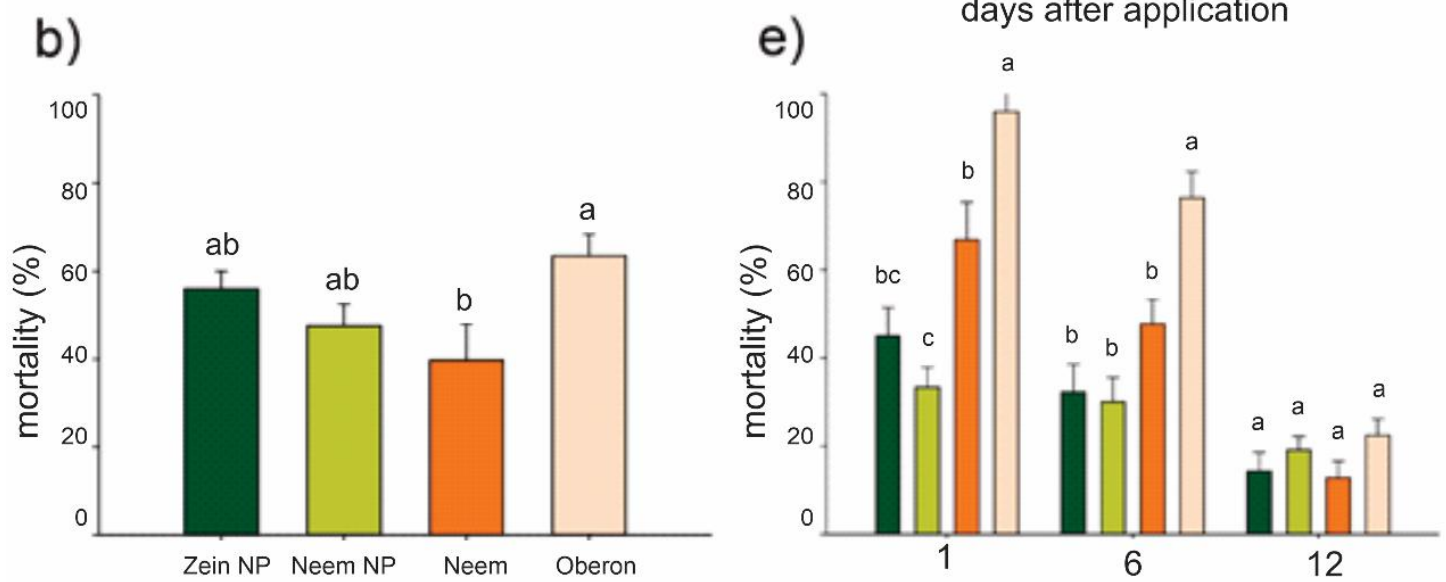

c)
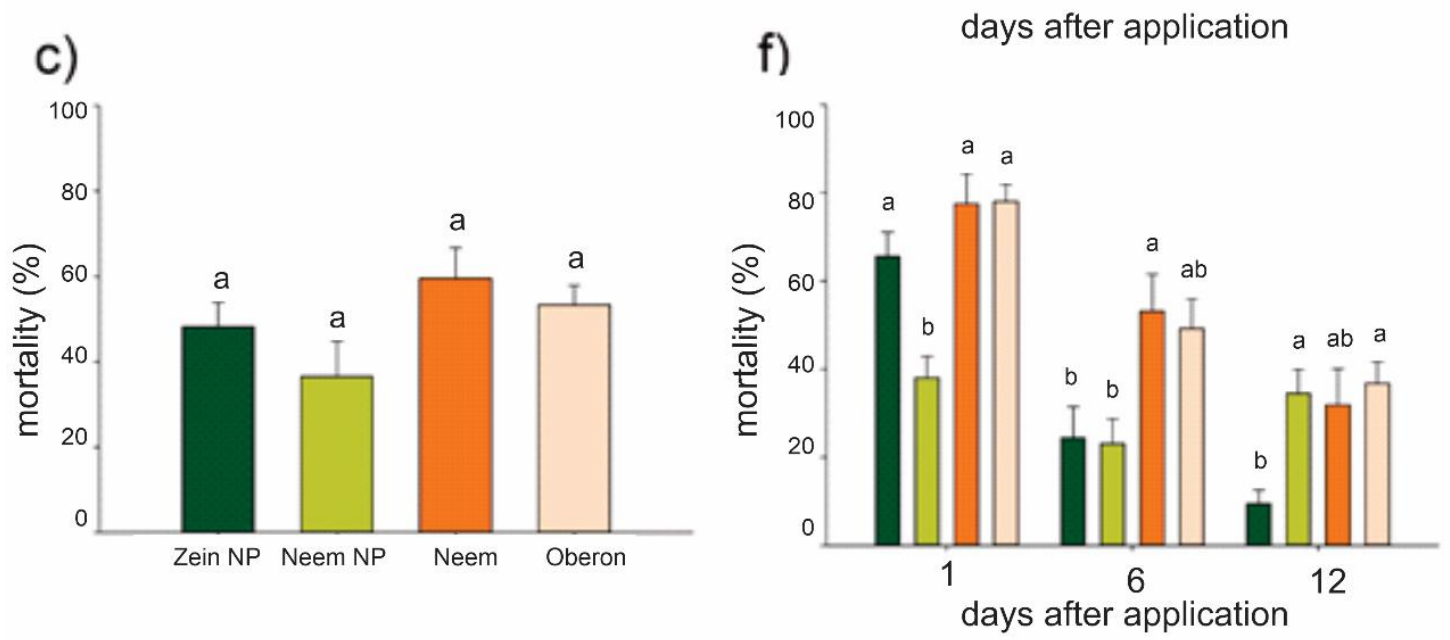

559 Fig. 5 Results of biological activity assays using Tetranychus urticae. Mortality 5

560 days after direct applications on the A) larvae, B) nymphs, and C) adults, using

561 zein nanoparticles (Zein NP), neem oil-loaded zein nanoparticles (Neem NP),

562 neem oil (Neem), and Oberon ${ }^{\circledR}$ (acaricide as positive control). Residual effects

563 on the D) larvae, E) nymphs, and F) adults analyzed on leaves collected 1, 2 and

5646 days after the application of the treatments. The data are expressed as the 
565 averages of eight repetitions $(n=8)$, normalized to \%. The error bars represent

566 the standard deviation. Different letters denote significant differences. A 567 significance level of $P<0.05$ was adopted using two-way ANOVA followed by the 568 Tukey post-hoc test.

\section{Discussion}

In relationship to the nanoparticle's characterization, the mean diameter obtained by DLS was higher than that obtained using NTA. A similar result was reported by Oliveira et al. (2018a) for zein nanoparticles. The encapsulation efficiency shows that novel zein nanocarrier systems have promising potential for the encapsulation and protection of active compounds. The negative potential zeta results were in agreement with the findings of Podaralla and Perumal (2012) and Oliveira et al. (2019), who used Pluronic F-68 to obtain zein nanoparticles

The physico-chemical stability results showed that although the nanoparticles in suspension presented oscillations of the mean diameter, the polydispersion and span indices remained similar to the values characteristic of stable formulations. The nanoparticle concentration also showed no significant alterations, while the encapsulation efficiency decreased, as expected since the nanocarrier released the active compound as a function of time. Nonetheless, despite the release, the loading still remained at $70 \%$, which could be considered high. Therefore, it could be concluded that the presence of Pluronic F-68 as a surfactant was effective in maintaining the stability of the nanobiopesticide.

Given that pest control would lead to plants being exposed to high concentrations of nanoformulations, the phytotoxicity of new nanotechnological 
588

589

590

591

592

593

594

595

596

597

598

599

600

601

602

603

604

605

606

607

608

609

610

611

612

systems should be carefully investigated (Yu et al. 2015). The photosynthetic activity and the growth of $P$. vulgaris plants were not affected by the nanoformulation, as well as it did not induce oxidative stress in plant cells. Taken together, these results indicated that this new nanobiopesticide is safe for application to $P$. vulgaris under the experimental conditions adopted. Our results corroborate the reports by Sridharan et al. (2015) and Oliveira et al. (2018a), which showed that neem oil and zein nanoparticles did not demonstrate phytotoxic potential, emerging as a tool for pest control in sustainable agriculture.

In contrast, this new nanobiopesticide increased insecticidal effects against store pest $A$. obtectus, which is one of the most important pests of $P$. vulgaris dry beans, multiplying in the field and post-harvest (Vuts et al. 2018). This insect has a wide variety of host plants and reduces the mass, volume, physiological quality, and germination index of beans, while increasing the temperature and water content, leading to losses of around 7-40\% (Mbogo et al. 2009). Bean producers and distributors control $A$. obtectus using insecticides including pyrethroids, organophosphates, and aluminum phosphide fumigant (Pimentel et al. 2012). However, the use of these compounds has led to concerns regarding environmental contamination, pest resistance evolution, and impacts on human health (Shelef et al. 2018; Pellegrini and Fernández 2018). Hence, this new technology for the control of $A$. obtectus that can contribute to safety in agriculture.

Also, the findings with the images of $A$. obtectus exposed to neem oilloaded zein nanoparticles labeled with rhodamine which show the nanobiopesticide in the ventral region, mouthpart and antennae open perspectives for improving understanding of the effects of nanoformulations. 
Using B. tabaci, another most serious polyphagous pests of field and

614 greenhouse crops, was observed the potential for using a lower concentration of

615 the neem oil to control whitefly. Different to the assay performed with $A$. obtectus

616 (which showed a directly proportional relationship between concentration

617 increase and insecticidal effect), a possible explanation for this result was that at

618 the lower concentration, the nanoparticles presented greater dispersion, which

619 reduced the possibility of aggregation and enhanced the capacity of the

620 nanoparticles to enter into contact with the organism, even penetrating its

621 integument.

An important point was that although the neem oil commercial product was recommended for use against this pest, the mortality shown was lower than expected (not reaching 50\%), which could have been due to the great ability of B. tabaci to develop resistance to pesticides. In addition, the different populations of $B$. tabaci present genetic differences that could be responsible for important biological differences among them, in terms of symbionts, feeding behavior, virus transmission, host plant variety, and resistance to insecticides (Harish et al. 2019; Hussain et al. 2019; Wang et al. 2019).

According to these results, Kumar (2008) reported mortality in B. tabaci using commercial neem oil (NeemAzal-U 17\%) under semi-field conditions and Boursier et al. (2011) found that neem plant extract had the same effect on whitefly as commercial neem oil. Campos et al. (2018a) and Oliveira et al. (2019) studied the effects of polymeric nanoparticle formulations containing essential oils against $H$. armigera and $C$. includens, respectively, and in both cases, a greater sublethal effect was obtained using the encapsulated compounds, compared to commercial compounds. On the other hand, Oliveira et al. (2018b) 
638 found that chitosan/gum arabic nanoparticles loaded with eugenol had an attractive effect for $B$. tabaci. It can be seen from these results that the effect of

640 the active agent can vary according to its form and the experimental conditions, which emphasizes the need to carry out an extensive evaluation of any new 642 system.

Finally, in relationship to $T$. urticae, considering that it is a pest that exhibits fast reproductive capacity and resistance to a wide range of active agents, this nanobiopesticide may be promising for field application, since it can confer protection of the active agent which led to prolonged effects and consequently reduce the need for reapplication of the product on the larvae, indicating the potential benefits of these nanotechnological products in agricultural applications. In the same way, Ahmadi et al. (2018) and Campos et al. (2018a) also showed the ability of nanoencapsulation to increase the acaricidal activities of natural compounds against T. urticae. neem oil showed good physicochemical stability during 90 days. It is important to emphasize that the encapsulation of the active compound significantly increased its effectiveness against the pest $A$. obtectus and fluorescence labeling of the nanoparticles enabled visualization of the interaction of the nanomaterial with the test organism. Besides, this new system had no phytotoxic effects on common bean plants under our experimental conditions and presented biological activity 659 against whitefly (B. tabaci) and two-spotted spider mite ( $T$. urticae). Therefore, 660 the present findings provide further support for the excellent potential of this nanobiopesticide to be used in pest control in sustainable agriculture. 


\section{Acknowledgments}

663 The authors are grateful for the financial support for this research provided by the

664 São Paulo Research Foundation (FAPESP, grants \#2017/21004-5, 665 \#2015/17120-4, and \#2013/11343-6) and the National Council for Scientific and 666 Technological Development (CNPq).

667 Conflicts of interest

668 There are no conflicts of interest to declare.

\section{Human and animal rights}

This article does not contain any studies with human participants or animals

671 (vertebrates) performed by any of the authors.

\section{References}

Ahmadi Z, Saber M, Akbari A, Mahdavinia GR (2018) Encapsulation of Satureja hortensis L. (Lamiaceae) in chitosan/TPP nanoparticles with enhanced acaricide activity against Tetranychus urticae Koch (Acari: Tetranychidae). Ecotoxicol Environ Saf 161:111-119. https://doi.org/10.1016/j.ecoenv.2018.05.051

Alexieva V, Sergiev I, Mapelli S, Karanov E (2001) The effect of drought and ultraviolet radiation on growth and stress markers in pea and wheat. Plant Cell Environ 24:1337-1344

Borgatta J, Ma C, Hudson-Smith N, et al (2018) Copper Based Nanomaterials Suppress Root Fungal Disease in Watermelon ( Citrullus lanatus ): Role of Particle Morphology, Composition and Dissolution Behavior. ACS Sustain Chem Eng 6:14847-14856. https://doi.org/10.1021/acssuschemeng.8b03379 
Boursier CM, Bosco D, Coulibaly A, Negre M (2011) Are traditional neem extract preparations as efficient as a commercial formulation of azadirachtin A? Crop Prot 30:318-322. https://doi.org/10.1016/j.cropro.2010.11.022

Camejo G, Wallin B, Enojärvi M (1998) Analysis of oxidation and antioxidants using microtiter plates. Free Radic Antioxid Protoc Methods Mol Biol 108:377-386

Campos EVR, Proença PLF, Oliveira JL, et al (2018) Carvacrol and linalool coloaded in $\beta$-cyclodextrin-grafted chitosan nanoparticles as sustainable biopesticide aiming pest control. Sci Rep 8:. https://doi.org/10.1038/s41598-018-26043-x

Corrêa AS, Pereira EJG, Cordeiro EMG, et al (2011) Insecticide resistance, mixture potentiation and fitness in populations of the maize weevil (Sitophilus zeamais). Crop Prot 30:1655-1666. https://doi.org/10.1016/j.cropro.2011.08.022

Fraceto LF, Grillo R, de Medeiros GA, et al (2016) Nanotechnology in Agriculture: Which Innovation Potential Does It Have? Front Environ Sci 4:. https://doi.org/10.3389/fenvs.2016.00020

Gott RC, Luo Y, Wang Q, Lamp WO (2014) Development of a biopolymer nanoparticle-based method of oral toxicity testing in aquatic invertebrates. Ecotoxicol Environ Saf 104:226-230. https://doi.org/10.1016/j.ecoenv.2014.03.017

Grillo R, Abhilash PC, Fraceto LF (2016) Nanotechnology Applied to BioEncapsulation of Pesticides. J Nanosci Nanotechnol 16:1231-1234. https://doi.org/10.1166/jnn.2016.12332

Grillo R, dos Santos NZP, Maruyama CR, et al (2012) Poly( $\varepsilon$ caprolactone)nanocapsules as carrier systems for herbicides: Physicochemical characterization and genotoxicity evaluation. J Hazard Mater 231-232:1-9. https://doi.org/10.1016/j.jhazmat.2012.06.019

Grillo R, Pereira AES, Nishisaka CS, et al (2014) Chitosan/tripolyphosphate nanoparticles loaded with paraquat herbicide: An environmentally safer alternative for weed control. J Hazard Mater 278:163-171. https://doi.org/10.1016/j.jhazmat.2014.05.079

Hamilton MA, Russo RC, Thurston RV (1977) Trimmed Spearman-Karber method for estimating median lethal concentrations in toxicity bioassays. Environ Sci Technol 11:714-719. https://doi.org/10.1021/es60130a004

Harish ER, K. M, T. M, et al (2019) Next generation sequencing reveals endosymbiont variability in cassava whitefly, Bemisia tabaci, across the agro-ecological zones of Kerala, India. Genome gen-2018-0050. https://doi.org/10.1139/gen-2018-0050 
Hasheminejad N, Khodaiyan F, Safari M (2019) Improving the antifungal activity of clove essential oil encapsulated by chitosan nanoparticles. Food Chem 275:113-122. https://doi.org/10.1016/j.foodchem.2018.09.085

Hoagland DR The water-culture method for growing plants without soil. 32

$\mathrm{Hu} \mathrm{K}$, McClements DJ (2014) Fabrication of surfactant-stabilized zein nanoparticles: A pH modulated antisolvent precipitation method. Food Res Int 64:329-335. https://doi.org/10.1016/j.foodres.2014.07.004

Hussain S, Farooq M, Malik HJ, et al (2019) Whole genome sequencing of Asia II 1 species of whitefly reveals that genes involved in virus transmission and insecticide resistance have genetic variances between Asia II 1 and MEAM1 species. BMC Genomics 20:507. https://doi.org/10.1186/s12864019-5877-9

Janković-Tomanić M, Jovanović DŠ, Savković U, et al (2015) Host expansion modifies activity of phosphatases in a legume store pest Acanthoscelides obtectus (Say). J Stored Prod Res 62:32-35. https://doi.org/10.1016/j.jspr.2015.03.008

Kamaraj C, Gandhi PR, Elango G, et al (2018) Novel and environmental friendly approach; Impact of Neem ( Azadirachta indica ) gum nano formulation (NGNF) on Helicoverpa armigera (Hub.) and Spodoptera litura (Fab.). Int J Biol Macromol 107:59-69. https://doi.org/10.1016/j.ijbiomac.2017.08.145

Koul O (2019) Nano-biopesticides today and future perspectives, 1st edition. Elsevier, Cambridge, MA

Kumar P (2008) Studies on loss of bio-efficacy of two indirect neem application over time (seed and soil) against Bemisia tabaci (Homoptera: Aleyrodidae) under semi-field conditions. J Asia-Pac Entomol 11:185-190. https://doi.org/10.1016/j.aspen.2008.08.003

Malaikozhundan B, Vaseeharan B, Vijayakumar S, Thangaraj MP (2017) Bacillus thuringiensis coated zinc oxide nanoparticle and its biopesticidal effects on the pulse beetle, Callosobruchus maculatus. J Photochem Photobiol B 174:306-314. https://doi.org/10.1016/j.jphotobiol.2017.08.014

Mbogo KP, Davis J, Myers JR (2009) Transfer of the ArcelinPhytohaemagglutinin-Ît Amylase Inhibitor Seed Protein Locus from Tepary bean (Phaseolus acutifolius A. Gray) to Common Bean (P. vulgaris L.). Biotechnology(Faisalabad) 8:285-295. https://doi.org/10.3923/biotech.2009.285.295

Mutungi C, Affognon HD, Njoroge AW, et al (2015) Triple-Layer Plastic Bags Protect Dry Common Beans ( Phaseolus vulgaris ) Against Damage by Acanthoscelides obtectus (Coleoptera: Chrysomelidae) During Storage. J Econ Entomol 108:2479-2488. https://doi.org/10.1093/jee/tov197 
Oliveira JL de, Campos EVR, Pereira AES, et al (2018a) Zein Nanoparticles as Eco-Friendly Carrier Systems for Botanical Repellents Aiming Sustainable Agriculture. J Agric Food Chem 66:1330-1340. https://doi.org/10.1021/acs.jafc.7b05552

Oliveira JL, Campos EVR, Germano-Costa T, et al (2019) Association of zein nanoparticles with botanical compounds for effective pest control systems. Pest Manag Sci 75:1855-1865. https://doi.org/10.1002/ps.5338

Oliveira JL, Campos EVR, Pereira AES, et al (2018b) Geraniol Encapsulated in Chitosan/Gum Arabic Nanoparticles: A Promising System for Pest Management in Sustainable Agriculture. J Agric Food Chem 66:53255334. https://doi.org/10.1021/acs.jafc.8b00331

Oliveira JL, Ramos Campos EV, Fraceto LF (2018c) Recent Developments and Challenges for Nanoscale Formulation of Botanical Pesticides for Use in Sustainable Agriculture. J Agric Food Chem 66:8898-8913. https://doi.org/10.1021/acs.jafc.8b03183

Pascoli M, Jacques MT, Agarrayua DA, et al (2019) Neem oil based nanopesticide as an environmentally-friendly formulation for applications in sustainable agriculture: An ecotoxicological perspective. Sci Total Environ 677:57-67. https://doi.org/10.1016/j.scitotenv.2019.04.345

Pascoli M, Lopes-Oliveira PJ, Fraceto LF, et al (2018) State of the art of polymeric nanoparticles as carrier systems with agricultural applications: a minireview. Energy Ecol Environ 3:137-148. https://doi.org/10.1007/s40974-018-0090-2

Pellegrini P, Fernández RJ (2018) Crop intensification, land use, and on-farm energy-use efficiency during the worldwide spread of the green revolution. Proc Natl Acad Sci 115:2335-2340. https://doi.org/10.1073/pnas.1717072115

Pimentel MAG, Faroni LRA, Corrêa AS, Guedes RNC (2012) Phosphine-induced walking response of the lesser grain borer (Rhyzopertha dominica). Pest Manag Sci 68:1368-1373. https://doi.org/10.1002/ps.3314

Podaralla S, Perumal O (2012) Influence of Formulation Factors on the Preparation of Zein Nanoparticles. AAPS PharmSciTech 13:919-927. https://doi.org/10.1208/s12249-012-9816-1

Prajitha N, Athira SS, Mohanan PV (2019) Bio-interactions and risks of engineered nanoparticles. Environ Res 172:98-108. https://doi.org/10.1016/j.envres.2019.02.003

Prasad R, Kumar V, Prasad SK (2014) Nanotechnology in sustainable agriculture: Present concerns and future aspects. Afr J Biotechnol 13:705713. https://doi.org/10.5897/AJBX2013.13554

Ross MA, Lembi CA (1985) Applied weed science. Burgess Pub. Co, Minneapolis, Minn. ISMB 978-0-8087-2958-7. 
Shelef O, Fernández-Bayo JD, Sher Y, et al (2018) Elucidating Local Food Production to Identify Principles and Challenges for Sustainable Agriculture. In: Sustainable Food Systems from Agriculture to Industry. Charis Galanakis, pp 47-81

Soares MA, Quintela ED, Mascarin GM, Arthurs SP (2014) Effect of temperature on the development and feeding behavior of Acanthoscelides obtectus (Chrysomelidae: Bruchinae) on dry bean (Phaseolus vulgaris L.). J Stored Prod Res 61:90-96. https://doi.org/10.1016/j.jspr.2014.12.005

Sridharan S, Shekhar KC, Ramakrishnan N (2015) Bioefficacy, Phytotoxicity, and Biosafety of Mineral Oil on Management of Whitefly in Okra. Int J Veg Sci 21:28-35. doi: 10.1080/19315260.2013.818607

Tofel KH, Kosma P, Stähler M, et al (2017) Insecticidal products from Azadirachta indica and Plectranthus glandulosus growing in Cameroon for the protection of stored cowpea and maize against their major insect pests. Ind Crops Prod 110:58-64. https://doi.org/10.1016/j.indcrop.2017.09.051

Verma ML (2017) Nanoscience in food and agriculture. 4: ... Springer, Cham

Viteri Jumbo LO, Faroni LRA, Oliveira EE, et al (2014) Potential use of clove and cinnamon essential oils to control the bean weevil, Acanthoscelides obtectus Say, in small storage units. Ind Crops Prod 56:27-34. https://doi.org/10.1016/j.indcrop.2014.02.038

Vuts J, Woodcock CM, König L, et al (2018) Host shift induces changes in mate choice of the seed predator Acanthoscelides obtectus via altered chemical signalling. PLOS ONE 13:e0206144. https://doi.org/10.1371/journal.pone.0206144

Yu Z, Sun X, Song H, et al (2015) Glutathione-Responsive Carboxymethyl Chitosan Nanoparticles for Controlled Release of Herbicides. MAS 06:591-604. doi: 10.4236/msa.2015.66062

Wang H-L, Lei T, Xia W-Q, et al (2019) Insight into the microbial world of Bemisia tabaci cryptic species complex and its relationships with its host. Sci Rep 9:6568. https://doi.org/10.1038/s41598-019-42793-8 